\title{
Long Range Polymer Chain Dynamics Studied by Fluorescence Quenching
}

\author{
Shiva Farhangi and Jean Duhamel* \\ Institute for Polymer Research, Waterloo Institute for Nanotechnology, Department of \\ Chemistry, University of Waterloo, 200 University Avenue West, Waterloo, ON N2L 3G1, \\ Canada
}

* To Whom correspondence should be addressed 


\begin{abstract}
Over the years, fluorescence quenching experiments have provided a robust analytical means to retrieve information about the internal dynamics of macromolecules in general and the Long Range Polymer Chain Dynamics (LRPCD) of linear chains in particular. This report reviews the results obtained to date with the two main fluorescence experiments based on collisional quenching that have been used over the years to describe LRPCD. These experiments involve the labeling of a chain with dyes and quenchers either at the ends of a monodisperse chain for fluorescence quenching end-to-end cyclization (fqEEC) experiments or randomly along a polydisperse chain for fluorescence decay analysis with the Fluorescence Blob Model (FBM). The advantages and disadvantages of these two types of experiments are discussed as well as their range of applications and applicability to the field of protein folding. In particular, this Perspective illustrates how fqEEc experiments are being applied to probe loop formation in polypeptides and how FBM analysis of randomly labeled polypeptides could help determine the size of foldons which are expected to solve Levinthal's long-standing paradox.
\end{abstract}

Keywords: Pyrene, fluorescence, excimer, chain dynamics, protein folding. 


\section{INTRODUCTION}

Dynamics and energetics continually oppose each other as a macromolecule folds towards its equilibrium conformation. In the case of an isolated linear chain having some structural units bearing associative pendants that can interact with each other, interactions between the pendants will induce the association of the structural units only if the dynamics of the main chain are sufficiently slow to allow enough contact time between the associative pendants to establish an interaction. In effect, if the kinetic energy of the structural units of a highly mobile chain is too large compared to the interaction energy between the associative pendants, this imbalance will prevent association. At the other extreme, despite the low kinetic energy of the structural units of a rigid polymer, these units will be unable to interact with each other due to the stiff backbone that prevents an unfolded macromolecule from changing its conformation and bringing the associative pendants into contact regardless of the strength of their interactions. Consequently, the fast and slow dynamics of highly flexible or rigid macromolecules bearing associative pendants are expected to prevent their intramolecular interactions and thus their association. In summary, interactions between the side chains of a polymer subject to its own internal dynamics will lead to a specific macromolecular conformation, and thus a specific macromolecular behavior in solution according to the balance that exists between dynamics and associative forces within the macromolecule.

There are many cases where these considerations apply, such as for the characterization of the viscoelastic properties of associative thickeners, ${ }^{1-6}$ but a much more challenging, and thus much more interesting, example aims to characterize the behavior of biological macromolecules such as proteins in solution. Compared to that of associative thickeners, the behaviour of proteins in solution is usually much more difficult to predict due to the infinite variation in protein 
sequences that leads to an infinite number of combinations of backbone dynamics and associative forces between side chains. For polypeptides, associative forces induced by the amino acid side chains and backbone dynamics depend on the nature of the amino acid substituents, the bulkiness of the substituents and their level of aggregation affecting the latter while the former depend on whether the substituents can interact via H-bonds, electrostatic repulsion or attraction, disulfur bridge formation, and hydrophobic association, to name but a few. Due to this inherent complexity, the characterization of the internal dynamics of biological macromolecules is a very active research area, in particular to determine the folding pathway of proteins. ${ }^{7-11}$ The overarching goal in these studies is to determine the time taken for the folding of structural intermediates toward the ultimate 3-dimensional structure of the protein, a process that would benefit from the characterization of the internal dynamics of individual polypeptide chains in solution. As a result, techniques capable of probing polymer chain dynamics in solution have attracted strong scientific interest. These include dynamic light scattering (DLS), ${ }^{12}$ nuclear magnetic resonance (NMR), ${ }^{13}$ electron spin resonance (ESR), ${ }^{14}$ rheology, ${ }^{15}$ optical tweezers, ${ }^{16}$ and various fluorescence-based techniques such as fluorescence microscopy, ${ }^{17}$ fluorescence anisotropy, ${ }^{18}$ or fluorescence dynamic quenching ${ }^{19-21}$ which includes fluorescence resonance energy transfer (FRET) and pyrene excimer formation.

Considering the immense range of experiments conducted to characterize different aspects of protein folding, it becomes important within the context of this Perspective to narrow down the field somewhat by differentiating between the experiments that aim to monitor the kinetics involved in the formation of folding intermediates along the folding pathway of native biomacromolecules in solution on the one hand, and those based on the study of synthetic polymers aiming to establish general physical principles for the characterization of polymer chain dynamics 
on the other hand. Since the kinetics involved during the folding of a polypeptide from a random coil into a structured protein usually span tens of microseconds to milliseconds ${ }^{22}$ while the chain dynamics of flexible polymers is typically probed in the submicrosecond time scale, ${ }^{19}$ this clear difference in time scales creates a well-defined boundary between the two research areas but obvious synergies exist between them. One case in point revolves around the use of macromolecules labeled at one specific location with a fluorophore and at another specific location with a quencher. The seminal experiment by Stryer and Haugland in 1967, where FRET was introduced as a Spectroscopic Ruler by attaching a fluorescent donor and acceptor to the ends of helix-forming oligopeptides with different degrees of polymerization (DP) to obtain the distance separating the two dyes, opened the path for applying FRET to determine the conformation of structured biological and synthetic macromolecules in solution. ${ }^{23}$ It took about ten more years to develop a theoretical framework that would extent the applicability of FRET to fluorescently endlabeled flexible oligopeptides to probe both the distance between the two chain ends and determine their translational diffusion coefficient $\left(D_{\text {trans }}\right){ }^{24}$

Beside FRET studies on end-labeled linear chains, Winnik showed in the early 1980s that the rate constant $<k_{1}>$ of intramolecular quenching of an excited fluorophore covalently attached to one end of a short monodisperse polystyrene and a quencher at the other end reflected quite accurately the expected dynamics of end-to-end cyclization (EEC) for polystyrene. ${ }^{19,} 25$ Fluorescence quenching EEC (fqEEC) experiments conducted by FRET or collisional quenching on fluorescently end-labeled oligopeptides provided a means to measure both $\left\langle k_{1}\right\rangle$ due to collisional quenching ${ }^{26}$ but also the average translational diffusion coefficient ( $\left.D_{\text {trans }}\right)$ and distance $\left(R_{\mathrm{F}-\mathrm{Q}}\right)$ between the dye and quencher from the FRET measurements. ${ }^{27}$ In turn, $R_{\mathrm{F}-\mathrm{Q}}$ could then be related to the conformation of the peptide, a larger $R_{\mathrm{F}-\mathrm{Q}}$ value indicating a more extended 
conformation. ${ }^{28}$ Similarly, excimer formation between pyrene molecules indicates that the pyrene labels have come within less than $1.0 \mathrm{~nm}$ from each other, ${ }^{29}$ which for pyrene-labeled macromolecules implies that a macromolecule that forms an excimer adopts a close conformation. In turn, cysteine mutagenesis of proteins enabled the covalent attachment of maleimide derivatives of dyes and quenchers onto cysteines that had been introduced at two specific positions in a protein. ${ }^{30}$ Fluorescence measurements on such fluorescently labeled proteins could then provide information about their conformation, ${ }^{31,32}$ and thus possible folding intermediates, in solution or inside living cells. ${ }^{28,30,33}$ Based on the above, it can be argued that the early fluorescence experiments to characterize the dynamics and end-to-end distance of fluorescently end-labeled synthetic short monodisperse polymers led to the design of proteins fluorescently labeled at specific positions whose conformation could be monitored along their folding pathway establishing a robust bridge between the former and latter research areas.

The present Perspective focuses on the latter research area by describing new developments in the use of time-resolved fluorescence for the characterization of polymer chain dynamics that leave aside the classic design of macromolecules fluorescently labeled at two, and only two, specific positions. Its purpose is four-fold. First, it will demonstrate that $<k_{1}>$ obtained with end-labeled polymers is unlikely to enable the characterization of the Long Range Polymer Chain Dynamics (LRPCD) of polymers that are both long and/or rigid such as proteins whose polypeptide backbone falls into this category. Second, it will reiterate the statement already made by this laboratory ${ }^{34}$ that long chains randomly labeled with dyes and quenchers are much better suited to study LRPCD than monodisperse oligomers labeled with a dye at one end and a quencher at the other end. This goes against the entrenched belief that short monodisperse polymers labeled at one end with a fluorophore and a quencher at the other end represent the sole experimental 
means to obtain $<k_{1}>$ quantitatively. As it turns out, the same quantitative information about polymer chain dynamics can be obtained by conducting time-resolved fluorescence experiments on actual polymers that are randomly labeled and whose fluorescence decays are analyzed with the Fluorescence Blob Model (FBM). ${ }^{35}$ Third, this Perspective proposes that the product $<k_{\mathrm{blob}} \times N_{\text {blob }}>$ obtained from the FBM analysis of decays acquired with randomly labeled polymers provides a measure of the Long Range Polymer Chain Dynamics (LRPCD) in solution that might be as robust as the glass transition temperature $\left(T_{\mathrm{g}}\right)$ is to describe LRPCD in the solid state. Since $T_{\mathrm{g}}$ represents the onset of polymer chain motion in the bulk which takes place according to a crankshaft mechanism involving a few tens of atoms, $T_{\mathrm{g}}$ can be viewed as a parameter that quantifies LRPCD in the bulk as the product $<k_{\text {blob }} \times N_{\text {blob }}>$ does for polymers in solution. ${ }^{36}$ Last but not least, since the FBM is based on the fact that a fluorescently labeled monomer inside the polymer coil can only probe the few monomers present in its direct vicinity that define a polymeric blob, it suggests that the FBM merely accounts for the inherent compartmentalization of the polymer coil into subdomains referred to as blobs. Interestingly, the blobs dealt with within the framework of the FBM could very well be the foldons invoked to describe the multistate folding of proteins among subdomains $\sim 20$ residues in size whose repeated folding and unfolding eventually leads to the 3D-structure of the folded protein. ${ }^{9,11}$ As proposed earlier, ${ }^{37}$ the inherent compartmentalization of a polymer coil into subdomains clearly demonstrated by the applicability of the FBM to the study of randomly labeled polymers might provide the rational for solving Levinthal's long standing paradox. ${ }^{38}$ Levinthal argued that proteins would take an impossibly long time to reach their most thermodynamically stable conformation if they tried to adopt their extremely large number of available conformations $(\Omega)$ one conformation at a time until they would find the most stable one. But since the extremely large $\Omega$ value is a consequence of its 
exponential dependency on the DP of a protein, itself a large number, the proposal that folding is controlled by a small subset of residues located within a blob or foldon implies that $\Omega$ depends exponentially on a fraction of DP, and is thus much smaller than originally suggested by Levinthal. If such a conclusion could be reached by applying the FBM to randomly labeled polymers, it would certainly be an exciting development. But before discussing in more details the four topics presented above, a brief review of the information obtained on the Long Range Polymer Chain Dynamics (LRPCD) retrieved from fqEEC experiments conducted on synthetic polymers or polypeptides is provided in the next two sections.

\section{END-TO-END CYCLIZATION OF PYRENE END-LABELED POLYMERS}

Over the past four decades, pyrene end-labeled monodisperse polymers have been instrumental in providing a quantitative measure of the rate constant of EEC, namely $\left\langle k_{1}>\right.$, by applying Birks' scheme shown in Scheme 1 to describe the excimer formation between the two pyrenyl endgroups. ${ }^{19,39}$ In this kinetic analysis, $<k_{1}>$ is a pseudo-unimolecular rate constant equal to the product of the bimolecular rate constant ( $k_{\text {diff }}$ ) of diffusive encounters between the two end groups times the local quencher concentration in the polymer coil equal to $1 / V_{\text {coil }}$ for end-labeled polymers. The brackets for $<k_{1}>$ are used to represent the average EEC rate constant due to the fact that even a monodisperse polymer has a molecular weight distribution with a small but finite width. Upon excitation, an excited pyrene can fluoresce with its natural lifetime $\tau_{\mathrm{M}}$ or encounter a ground-state pyrene to form an excimer $\left(E 0^{*}\right)$. The excimer can then fluoresce with a lifetime $\tau_{\mathrm{E} 0}$ or dissociate with a rate constant $k_{-1}$ that is small with respect to $\tau_{\mathrm{E} 0} 0^{-1}$ at temperatures lower than $35{ }^{\circ} \mathrm{C}$ for pyrene. As a result, $k_{-1}$ is often neglected and is not shown in Scheme 1.

The importance of neglecting excimer dissociation in Scheme 1 must be recognized. Fluorescence experiments on fluorescently labeled macromolecules assume that the excited dye is 
surrounded by quenchers whose spatial distribution reflects the conformation and dynamics of the macromolecule. If dissociation is important however, each dissociative event results in quenchers being located right next to an excited dye and the spatial distribution of quenchers no longer reflects the conformation of the macromolecule. Thus the ability to neglect dissociation, as has been firmly established experimentally for pyrene excimer formation, ${ }^{19,25,34,39,40}$ ensures that the spatial distribution of quenchers is truly representative of the conformation adopted by the macromolecule. These considerations have been discussed in a recent review. ${ }^{20}$

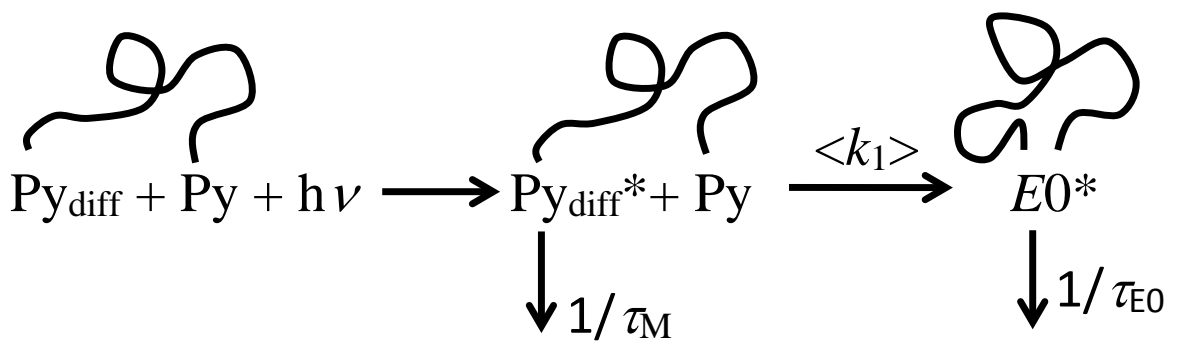

Scheme 1. Birks' scheme used to describe pyrene excimer formation for end-labeled monodisperse linear chains. ${ }^{19,39}$

In successive studies on different types of pyrene end-capped monodisperse polymers, the Winnik group established that the rate constant $<k_{1}>$ of intramolecular excimer formation between one excited pyrene and one ground-state pyrene scaled as $N^{-\gamma}$ where $N$ represented the number average chain length spanning the two pyrenes taken as the total number of atoms in the polymer chain and in the spacer linking pyrene to the main chain. For longer chains where the spacer can be neglected, the degree of polymerization (DP) of vinyl polymers approaches $N / 2$. The log-log plot of $\left\langle k_{1}>\right.$-vs- $N$ in Figure 1 showed that $<k_{1}>$ for polystyrene in cyclohexane at $34.5^{\circ} \mathrm{C}$, a $\theta$-solvent for PS, scaled as $N^{-1.62} \cdot{ }^{19,25}$ Considering that $<k_{1}>$ is a pseudo-unimolecular rate constant equal to the product $k_{\text {diff }} \times[P y]_{\text {loc }}$ where $k_{\text {diff }}$ is the bimolecular rate constant of excimer formation 
and $[P y]_{\text {loc }}$ is the concentration equivalent to one pyrene inside the polymer coil ([Py $\left.]_{\text {loc }}=1 / V_{\text {coil }}\right)$, the exponent $\gamma$ of 1.62 found for the relationship $<k_{1}>\propto N^{-1.62}$ suggested that the polymer coil radius $\left(R_{\text {coil }}\right)$ scaled as $N^{0.54}(0.54=1.62 / 3)$, a $\gamma$ value close to the Flory exponent of 0.50 expected for a polymer in a $\theta$-solvent.

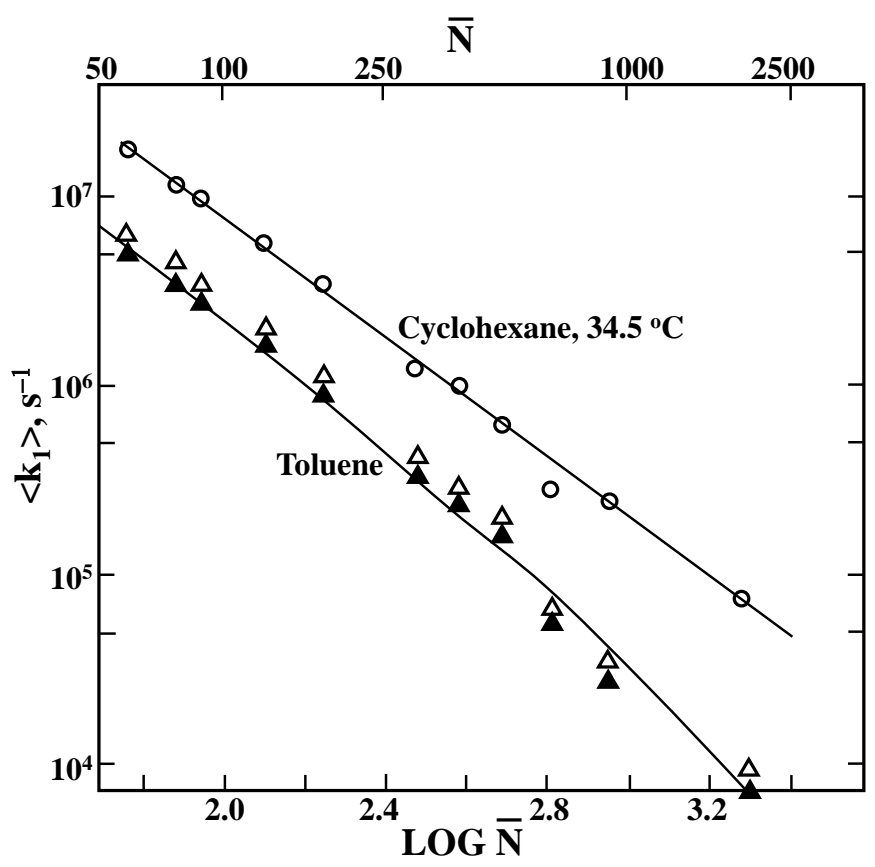

Figure 1. Log-log plots of the rate constant $<k_{1}>$ of pyrene excimer formation vs. the number of chain atoms $N$ of Py2-PS constructs in $(\bigcirc)$ cyclohexane at $34.5^{\circ} \mathrm{C} ;(\triangle)$ toluene at $22^{\circ} \mathrm{C} ;(\mathbf{\Delta})<k_{1}>$ values in toluene adjusted to the viscosity of toluene at $34.5^{\circ} \mathrm{C} .{ }^{19}$

The implication of the $<k_{1}>\propto N^{-1.62}$ relationship was that $<k_{1}>$ for longer Py2-PS constructs became vanishingly small and harder to measure accurately from the analysis of fluorescence decays alone. One solution proposed by the Winnik group to this problem was to use the proportionality that exists for shorter pyrene end-labeled polymers between the ratio $I_{\mathrm{E}} / I_{\mathrm{M}}$ of the fluorescence intensity of the excimer $\left(I_{\mathrm{E}}\right)$ over that of the monomer $\left(I_{\mathrm{M}}\right)$ obtained from the analysis 
of the fluorescence spectra and $<k_{1}>$ where $<k_{1}>$ could be measured accurately from the analysis of the fluorescence decays. The determination of the proportionality constant that exists between $I_{\mathrm{E}} / I_{\mathrm{M}}$ and $<k_{1}>$ for short chains enabled the Winnik group to predict the $<k_{1}>$ value of longer chains based on their $I_{\mathrm{E}} / I_{\mathrm{M}}$ ratio. $^{19,25}$ This approximation was used to build the plot shown in Figure 1 for $N$ values larger than about $600(\mathrm{DP} \sim 300)$ in cyclohexane at $34.5^{\circ} \mathrm{C}$ or about 250 (DP 125) in toluene at $22{ }^{\circ} \mathrm{C}$ corresponding to a $<k_{1}>$ value of $\sim 5 \times 10^{5} \mathrm{~s}^{-1}$. Toluene being a good solvent for PS resulted in a larger average separation distance between the pyrene end groups which led to less excimer formation, making it more challenging to measure $<k_{1}>$ for long $\mathrm{Py}_{2}$-PS constructs than in cyclohexane at $34.5^{\circ} \mathrm{C}$, a $\theta$-solvent for PS. These experiments were rapidly expanded to other polymers such as pyrene end-labeled poly(ethylene oxide) (Py2-PEO), ${ }^{40}$ poly(dimethyl siloxane) (Py2-PDMS), ${ }^{41}$ and polycarbonate (Py2-PC). ${ }^{42}$ As it turned out, the experiments carried out on pyrene end-labeled synthetic polymers to gain information about their LRPCD could also be readily applied to peptides as described hereafter.

\section{LOOP FORMATION DURING PROTEIN FOLDING}

As alluded to earlier, the quasi infinite number of peptide sequences that can be generated from the 20 most common amino acids makes it particularly challenging to predict the LRPCD of proteins which depend on the size of the substituent of each amino acid. In this context, fqEEC experiments can provide some valuable dynamic information on the backbone flexibility of any given peptide sequence. In particular, fqEEC experiments already provide information about the folding pathway of the most elementary structural element found in a protein, namely that of loop formation. ${ }^{43,44}$

Fluorescence quenching EEC experiments conducted on oligopeptides are based on the same principles that were first introduced in this review for pyrene end-labeled linear polymers, ${ }^{19}$ 
namely attaching a luminophore which can either fluoresce or phosphoresce at one end of the chain and an appropriate quencher at the other end and measuring the rate constant for luminescence quenching EEC (lqEEC). The luminophore can be quenched on contact either by fluorescence ${ }^{26,45-}$ ${ }^{48}$ or phosphorescence ${ }^{49,50}$ dynamic quenching or triplet-triplet energy transfer (TTET) $)^{43,44,51-57}$ or through space by fluorescence resonance energy transfer $\left(\right.$ FRET) ${ }^{27,57-60}$ or electron transfer through a Dexter mechanism. ${ }^{61,62}$ As for synthetic polymers, some lqEEC experiments conducted on short end-labeled peptides yielded the same $\left\langle k_{1}>\propto N^{-\gamma}\right.$ scaling law reported previously for synthetic polymers. The kinetics involved in the quenching on contact in a lqEEC experiment can be handled by Scheme 1 introduced earlier by using the dye and the quencher in lieu of the excited and ground-state pyrene, respectively. Quenching processes occurring over a distance by a FRET or a Dexter mechanism are summarized in Scheme 2 where $D$ and $A$ are the energy donor and acceptor, respectively. The resemblance between Schemes 1 and 2 is striking.

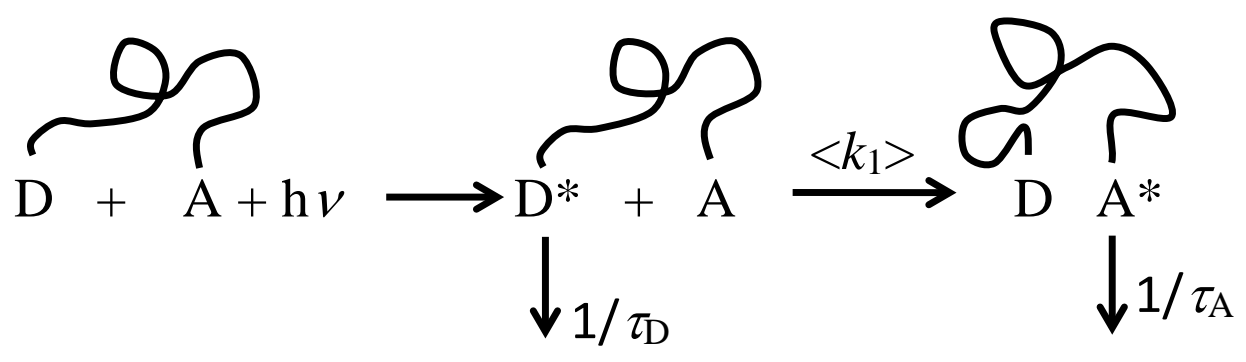

Scheme 2. Kinetic scheme representing the quenching of an energy donor by an acceptor over distance for end-labeled monodisperse linear chains.

3.A. Dynamic fluorescence quenching: In fqEEC experiments, $<k_{1}>$ for oligopeptides is influenced by the amino acid sequence and the length of the chain since a more rigid or longer peptide results in a smaller $<k_{1}>$ value. The effect of chain length on $<k_{1}>$ was investigated for the oligopeptide Trp-(Gly-Ser)n-DBO-NH $2\left(n=0,1,2,4,6\right.$, and 10). ${ }^{46}$ In these experiments, the fluorophore was 2,3-diazobicylo [(2.2.2)] oct-2ene (DBO) and tryptophane (Trp) was the quencher. The quenching 
of $\mathrm{DBO}$ by $\operatorname{Trp}$ in water at $23^{\circ} \mathrm{C}$ was monitored by time-resolved fluorescence. $<k_{1}>$ was determined and found to decrease continuously from 4.1 to $1.1 \times 10^{7} \mathrm{~s}^{-1}$ when the oligopeptide length was increased from $n=0$ to 10 approaching the expected trend $<k_{1}>\propto N^{-1.5}$ for the longest peptides. Similar experiments were carried out with a series of Oxa-(Gly-Ser $)_{\mathrm{n}}$-Trp $(n=2-15)$ where the oxazine (Oxa) dye was quenched by $\operatorname{Trp}^{43}$ For the longer peptides, the scaling relationship $<k_{1}>\propto N^{-1.4}$ was obtained in quite good agreement with the previous study.

3.B. Triplet-Triplet Energy Transfer (TTET): TTET takes place upon contact between a triplet donor and a triplet acceptor. These experiments take advantage of the long lifetime of the triplet donor which can reach milliseconds, thus providing a long time window to probe even the slowest dynamic processes encountered in the stiffest macromolecules. However these long-lived dyes are also more prone to adventitious quenching by impurities in the solvent. Despite these experimental challenges, this technique has been employed to measure the loop formation rate constant of peptides. In these experiments, xanthane (Xan) is often used as a triplet donor and naphthalene (N) as acceptor. ${ }^{52,53,55}$ The intrachain diffusion contact in Xan-(Gly-Ser)n-NAla-Gly-Ser sample with $n=1-28$ could be characterized by monitoring the xanthane triplet absorbance band at $590 \mathrm{~nm}$ whose decay analysis yielded the lqEEC rate constant $<k_{1}>$ which was found to decrease as $N^{-1.7} .53$

The dependency of $<k_{1}>$ on peptide length has also been reported in other studies, in particular for a series of peptides that was prepared with Trp at one end and Cys at the other for the peptidic constructs Cys-(Ala-Gly-Gln)n-Trp with $n$ taking values between 1 and $6 .{ }^{49}$ Upon excitation of Trp into the triplet state, the excited Trp could transfer its excess energy to Cys upon contact by TTET with a rate constant $<k_{1}>$ that was plotted in Figure 2 as a function of peptide length. The expected scaling relationship $<k_{1}>\propto N^{-1.5}$ in a $\theta$-solvent was only observed for the longest peptides with 19 peptide bonds. ${ }^{49}$ The fact that a sufficiently large degree of polymerization 
(DP ${ }_{\mathrm{ON}}$ ) must be reached before the $<k_{1}>\propto N^{-\gamma}$ relationship is being obeyed is a general observation.

Figure 2 also demonstrates that the $<k_{1}>$ value obtained for short oligomers is not representative of the LRPCD of long polypeptides which would be akin to full length proteins. The importance of DPon as the minimum DP above which the $<k_{1}>\propto N^{-\gamma}$ relationship holds will be discussed in more detail at a later stage of this Perspective.

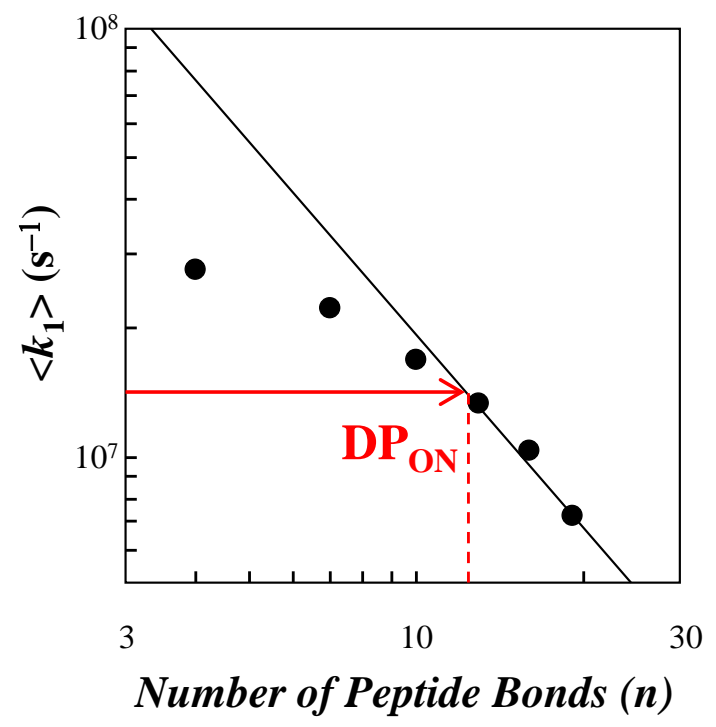

Figure 2. Dependence of the intramolecular quenching rate constant on peptide length. ${ }^{49}$ "Copyright (2000) National Academy of Sciences, U.S.A."

3.C. Fluorescence Resonance Energy Transfer (FRET): FRET has been applied to probe peptide chain dynamics by labeling the chain ends with a fluorescence donor and acceptor. The efficiency of FRET depends strongly on the distance between the donor and the acceptor molecule and this effect can be employed to probe the diffusive encounters between the two ends. At the initial time, the donor-acceptor pairs separated by a short distance undergo much more efficient FRET than those separated by a long distance. The depletion of donor-acceptor pairs separated by a short distance at early times creates a diffusion sink whereby D-A pairs separated by a large distance 
will use diffusion to reduce their average distance. Since the entire process takes place over a period of time that is measurable by time-resolved fluorescence, the fluorescence decay can be analyzed to yield the rate constant for quenching by FRET ( $\left.k_{\mathrm{ET}}\right)$ which depends on the flexibility of the chain, a more flexible chain resulting in a larger $k_{\mathrm{ET}}{ }^{51}$ Analysis of the fluorescence decays yields the distance distribution separating the D-A pairs and the diffusion coefficient reflecting the mobility of the dyes and thus the flexibility of the chains. A more recent application of the FRET principles has led to the development of single-molecule FRET (smFRET) experiments which have focused mainly on the characterization of the internal dynamics and conformation (via endto-end distance analysis) of intrinsically disordered proteins (IDPs). The following section presents some selected examples of FRET applications but by no means constitutes a complete description of the field. Readers interested in developing a deeper understanding of the study of macromolecules by FRET and smFRET are directed to recent reviews that are dedicated to this research topic. $^{28,30,51,63}$

The main application of FRET to characterize macromolecules is to determine the molecular distance spanning an energy donor and acceptor covalently attached at two specific locations of a same macromolecule. This information can then be applied to predict the conformation of the macromolecule, either as an expanded polymer coil or a collapsed structured protein. Furthermore, the FRET efficiency can also be monitored by changing solution conditions to probe the effect of these changes on the conformation of the macromolecule of interest. For instance, addition of polymers such as poly(ethylene glycol) to an aqueous solution of fluorescently labeled IDPs can result in their compression by building up the osmotic pressure of the solution thus mimicking the crowding experienced by the proteins inside a cell. ${ }^{64}$ Old results have also been revisited and their conclusions refined thanks to the availability of much more 
sensitive instrumentation. The FRET efficiency and end-to-end distances have been re-measured for the polyproline (PP) helices originally used by Stryer and Haugland to demonstrate that FRET can be applied as a spectroscopic ruler. ${ }^{23,65}$ In fact, the assumption that the dyes can be viewed as point-dipoles was found to no longer hold for very short PP helices resulting in less efficient FRET whereas longer PP helices underwent some bending that resulted in more efficient FRET. ${ }^{65}$ Yet earlier work showed that intramolecular distances between dyes covalently attached to the ends of short PP strands could be reliably retrieved by using a D-A pair having a small $R_{\mathrm{o}}$ value of $10 \AA .^{58}$ Intriguing results have been also reported by applying FRET to monitor the end-to-end distance of charged short oligopeptides. ${ }^{59}$ Whereas an hexaarginine or hexahistidine showed a collapse of its molecular dimension at high $\mathrm{pH}$, a decrease in $\mathrm{pH}$ from 12 to 1 did not affect the molecular dimension of hexa(aspartic acid) and hexa(glutamic acid), contrary to what would have been expected both intuitively and from molecular dynamics simulations. ${ }^{60}$

Beside conformational studies, FRET has also been applied to characterize the internal dynamics of fluorescently end-labeled short peptides. The FRET efficiency between the excited Trp and the ground-state DBO was investigated for a series of oligopeptides Trp-(Pro) ${ }_{\mathrm{n}}-\mathrm{DBO}-\mathrm{NH}_{2}$ ( $n=1,2,4$, and 6$)$ by steady-state and time-resolved fluorescence. ${ }^{58}$ The fluorescence intensity and average decay time of Trp were found to increase with increasing chain length, a clear indication of reduced FRET with increasing chain length but the small number and short length of the samples investigated did not allow the author to provide a scaling relationship between $k_{\mathrm{ET}}$ and $N$.

\section{SUITABILITY OF FLUORESCENCE EEC EXPERIMENTS TO PROBE LRPCD}


Despite their intensive use to probe the LRPCD of numerous synthetic and biological polymers in solution and the claim made since their inception in the late 1970s that fqEEC experiments can provide quantitative information on the magnitude of LRPCD in solution, ${ }^{19}$ it is fair to state that to date, these experiments have failed to deliver a unique parameter like $T_{\mathrm{g}}$, the glass transition temperature for polymers in the bulk, that would allow experimentalists to gauge the magnitude of the LRPCD of a polymer of interest in solution. Furthermore, as this survey of the current literature on fqEEC experiments has highlighted, fqEEC experiments are never applied to "real" polymers in solution with a degree of polymerization much greater than 100 , the only exceptions in the literature being the study on $\mathrm{Py}_{2}$-PS described in Figure $1^{19}$ and triplet-triplet absorption measurements enabled by a much longer-lived dye ${ }^{66}$ The main reason for this state of affair could be rationalized after the fact for polymers such as $\mathrm{PS}^{67}$ or poly(butyl methacrylate) ${ }^{68}$ (PBMA) in tetrahydrofuran where the pyrene label was randomly incorporated into the chain via copolymerization of styrene or butyl methacrylate with 1-pyrenebutyl acrylate or methacrylate, respectively. For these samples whose fluorescence decays were analyzed according to the Fluorescence Blob Model (FBM), pyrene excimer formation was found to occur locally within a subvolume of the polymer coil referred to as a blob made of about 50 monomers corresponding to the volume probed by an excited pyrene. This insight led to the unavoidable conclusion that LRPCD would not enable diffusive encounters between an excited and a ground-state pyrene covalently attached to the ends of a PS or PBMA chain made of many more than two blobs or 100 structural units. In turn, this observation explained why hardly any excimer could be detected in cyclohexane at $34.5^{\circ} \mathrm{C}$ and toluene at $22{ }^{\circ} \mathrm{C}$ for Pyz-PS constructs with a degree of polymerization (DP) greater than $100 .{ }^{19,25}$ Past a critical degree of polymerization ( $\mathrm{DP}_{\text {crit }}$ ), a fraction of the end- 
labeled chains have an end-to-end distance that is too large for an excited and a ground-state pyrene to encounter and form an excimer while one of the two pyrenes remains excited.

Figure 3 illustrates the concept of $\mathrm{DP}_{\text {crit. }}{ }^{69}$ As long as the volume probed by the excited pyrene, which is referred to as a blob, is larger than the polymer coil volume, the excited pyrene at one end of the chain will encounter the ground-state pyrene at the other end and form an excimer. This situation corresponds to Figure 3A. Under such conditions, the molar fraction ( $\left.f_{\text {free }}\right)$ of pyrene monomers that do not form excimer and behave as if they were free in solution equals zero since all excited pyrenes lead to excimer formation and the kinetics of excimer formation between the two pyrene end-labels applies. ${ }^{19}$ But as the chain length increases, $\mathrm{DP}_{\text {crit }}$ is reached where the blob becomes smaller than the polymer coil. As illustrated in Figure 3B, a fraction of the chains have their ground-state pyrene located outside the blob preventing excimer formation and yielding nonzero free values. Birks’ scheme described in Scheme 1 no longer applies.

Of course, $\mathrm{DP}_{\text {crit }}$ where diffusive encounters between the two ends of a linear chain are no longer possible depends on several parameters. $\mathrm{DP}_{\text {crit }}$ is expected to increase with lower solvent viscosity, poorer solvent quality toward the polymer, greater backbone flexibility, and longer lifetime of the dye. Evidence of the existence of $\mathrm{DP}_{\text {crit }}$ was found when the effect of solvent viscosity on EEC was investigated with a series of Py2-PEO samples. ${ }^{69}$ The fraction of pyrene monomers that did not form any excimer and emitted as if they were free in solution ( $\left.f_{\text {free}}\right)$ due to their location outside of a blob was found to increase linearly with increasing solvent viscosity for a given $\mathrm{Py}_{2}$-PEO sample as well as increasing polymer molecular weight in a given solvent, both effects contributing to hindering the encounters between an excited and a ground-state pyrene. In dioxane, an organic solvent with a viscosity $(\eta)$ of $1.37 \mathrm{mPa} . \mathrm{s}$, an $f_{\text {free }}$ value of 0.85 was obtained for Py2-PEO(10K), a 10K monodisperse PEO end-capped with pyrene, indicating that hardly any 
excimer was produced under these conditions. By comparison, the same $\mathrm{Py}_{2}-\mathrm{PEO}(10 \mathrm{~K})$ sample yielded an $f_{\text {free }}$ value of only 0.11 in acetone ( $\eta=0.32 \mathrm{mPa} . \mathrm{s}$ ) indicating that $89 \%$ of the pyrene labels formed excimer in that solvent where the lower viscosity allowed more encounters between the pyrene terminals. ${ }^{69}$ This result supported the conclusion reached earlier that fqEEC experiments are better applied to oligomers rather than polymers. ${ }^{34}$ For longer chains whose

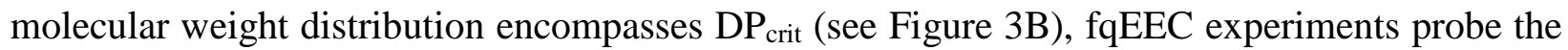
distribution of chains whose degree of polymerization is larger or smaller than $\mathrm{DP}_{\text {crit }}$ and no longer reflects the kinetics of EEC.

Since information retrieved on LRPCD is questionable for long end-labeled chains with a degree of polymerization greater than $\mathrm{DP}_{\text {crit, }}$ fqEEC experiments should focus instead on shorter chains where a strong fluorescence quenching reflects efficient EEC as is usually being done in the literature. One problem that arises from this strategy however is that, as discussed in Section 3.B, the oligomers must have a DP larger than DPon for the kinetics of fqEEC to follow the scaling behaviour between $<k_{1}>$ and chain length as was observed in Figure 2 for a series of -(Ala-GlyGln $)_{n}-$ oligopeptides ${ }^{49}$ and in another report. ${ }^{46}$ Consequently, DP must be shorter than $\mathrm{DP}_{\text {crit }}$ to ensure that the polymer coil fits inside a blob (see Figure 3A) but larger than $\mathrm{DP}_{\mathrm{ON}}$ characterizing the onset where the scaling relationship $\left\langle k_{1}>\sim N^{-\gamma}\right.$ is obeyed. For this reason, every fqEEC experiment requires the preparation of several end-labeled oligomeric constructs with increasing chain length whose EEC kinetics need to be characterized to establish the $\mathrm{DP}_{\mathrm{ON}}-\mathrm{DP}_{\text {crit }}$ range where the $k_{\mathrm{cy}} \sim N^{-\gamma}$ relationship holds. 

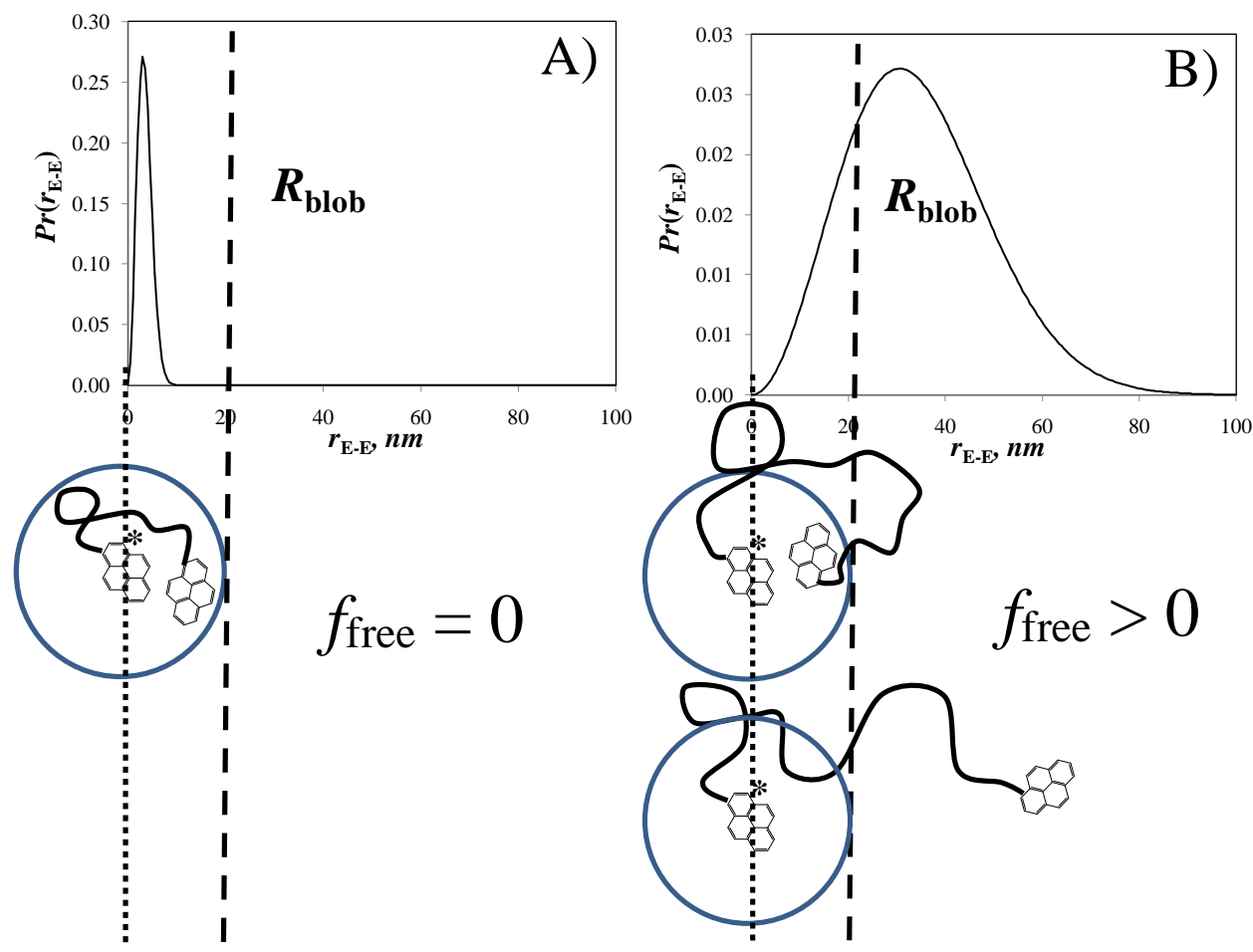

Figure 3. Dependency of $f_{\text {free }}$ as a function of $r_{\mathrm{EE}} / R_{\mathrm{blob}}$. A) $r_{\mathrm{EE}} / R_{\mathrm{blob}}<<1$ and $f_{\text {free }}=0$. B) $r_{\mathrm{EE}} / R_{\mathrm{blob}}>1$ and $f_{\text {free }}>0 .{ }^{69}$ The vertical dashed line represents the $r_{\mathrm{EE}}$ value corresponding to $\mathrm{DP}_{\text {crit. }}$.

In turn, a comparison of the LRPCD of different polymers based on their EEC kinetics requires that their respective $\mathrm{DP}_{\mathrm{ON}}-\mathrm{DP}_{\text {crit }}$ range overlap. The extent of overlap can be visualized in a plot of $<k_{1}>$ as a function of DP for different polymers as illustrated in Figure 4 for different series of $\mathrm{Py}_{2}-\mathrm{PS},{ }^{19,25,34} \mathrm{Py}_{2}-\mathrm{PEO},{ }^{69}$ and $\mathrm{Py}_{2}-\mathrm{PDMS}^{41}$ samples in toluene. Using a similar solvent eliminates differences in solvent viscosity although solvent quality toward the polymer might play a role. 


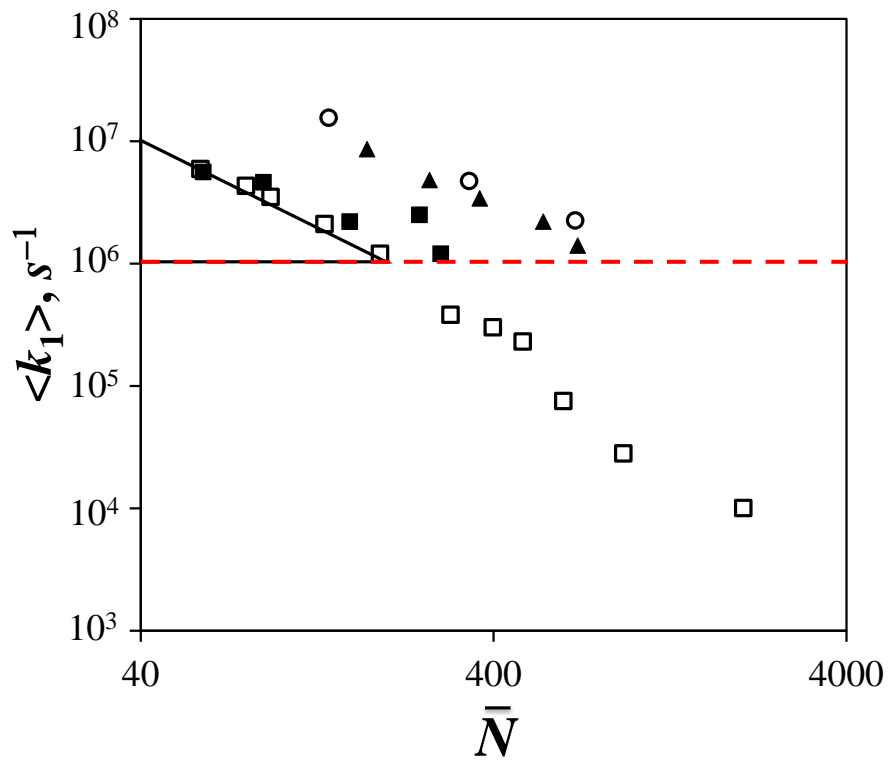

Figure 4. Plot of $<k_{1}>$ vs. $N$ in toluene for ( $\square$, Winnik) $\mathrm{Py}_{2}-\mathrm{PS}$ at $22{ }^{\circ} \mathrm{C},{ }^{19}$ ( $\square$, Ingratta et al.) $\mathrm{Py}_{2}-$ $\mathrm{PS},{ }^{34}$ (O, Chen et al.) Py2-PEO, ${ }^{69}$ and ( $\boldsymbol{\Delta}$, Svirskaya et al.) Py2-PDMS.$^{41}$ The dashed line indicates the limit below which very little excimer is being formed.

Similar trends were obtained for short Py2-PS samples in toluene by Winnik ${ }^{19}$ and Ingratta et al. ${ }^{34}$ Wider differences in $<k_{1}>$ were observed for longer Py2-PS chains due to differences in the analysis of the fluorescence decays, the analysis being based either on the fit of the monomer fluorescence decays or the equivalence between the $I_{\mathrm{E}} / I_{\mathrm{M}}$ ratio and $<k_{1}>$ in the Winnik study ${ }^{19}$ while Ingratta et al. ${ }^{34}$ applied solely global analysis of the monomer and excimer decays to retrieve $<k_{1}>$. Based on the trends shown in Figure 4, the $\mathrm{Py}_{2}-\mathrm{PS}$ series yielded the slowest LRPCD whereas the $\mathrm{Py}_{2}$-PEO series yielded the fastest. For a similar chain length, the $<k_{1}>$ values obtained for the Py2-PEO series were only slightly higher than those of $\mathrm{Py}_{2}$-PDMS. These trends are reasonable based on $T_{\mathrm{g}}$ values which reflect the flexibility of a polymer based on its free volume in the bulk. Indeed, $T_{\mathrm{g}}$ has been reported to equal $100{ }^{\circ} \mathrm{C}$, between -115 and $-50{ }^{\circ} \mathrm{C}$, and $-123{ }^{\circ} \mathrm{C}$ for PS, PEO, and PDMS, respectively. ${ }^{70}$ 
Rapid visual inspection of Figure 4 indicates that, except for the study by Winnik for the Py2-PS series in toluene that reports $<k_{1}>$ values as low as $10^{4} \mathrm{~s}^{-1}$ and extends up to $N$ values as large as $2,000,{ }^{19}$ all other trends report $<k_{1}>$ values that are never lower than $10^{6} \mathrm{~s}^{-1}$ which corresponds to the threshold below which, excimer fluorescence becomes barely detectable in a steady-state fluorescence spectrum, certainly a consequence of the DP becoming larger than $\mathrm{DP}_{\text {crit. }}$ The reason for the unusual range in $N$ values covered by the Winnik study ${ }^{19}$ has been discussed earlier in Section 2 and is due to the use of the $I_{\mathrm{E}} / I_{\mathrm{M}}$ ratio to predict the small $<k_{1}>$ values obtained for the longer chains. Yet the validity of this procedure has been questioned (see Figure 3). ${ }^{69}$ Consequently, the $<k_{1}>$-vs- $N$ trends shown in Figure 4 suggest that for pyrene end-labeled synthetic polymers, fqEEC experiments provide reliable $<k_{1}>$ values as long as $<k_{1}>$ is greater than $10^{6} \mathrm{~s}^{-1}$ corresponding to $\mathrm{DP}_{\text {crit }}$ values of $N / 2=90$ (Winnik) $)^{19}$ or 170 (Duhamel) ${ }^{34}$ for Py2-PS, $N / 3=430(\text { Duhamel })^{69}$ for Py 2 -PEO, and N/2 $=470(\text { Winnik })^{41}$ for Py2-PDMS. As mentioned earlier, differences in the $\mathrm{DP}_{\text {crit }}$ values that correspond to $<k_{1}>$ values obtained for large DPs are mostly due to differences in the analysis of the fluorescence decays. But regardless of these differences and after accounting for the number of chain atoms found in each structural unit of the different polymers, the $\mathrm{DP}_{\text {crit }}$ values retrieved from the trends shown in Figure 4 follow the sequence PS $<$ PEO $\leq$ PDMS in toluene, a sequence that reflects their expected chain flexibility based on their $T_{\mathrm{g}}$.

The $<k_{1}>$-vs- $N$ trend obtained with the Py2-PS series in Figure 4 represents an interesting case as polystyrene exhibits the slowest LRPCD among the $\mathrm{Py}_{2}-\mathrm{PS}, \mathrm{Py}_{2}-\mathrm{PEO}$, and $\mathrm{Py}_{2}-\mathrm{PDMS}$ series. For the Py2-PS in toluene, $\mathrm{DP}_{\text {crit }}$ values of 90 and 170 were retrieved representing $M_{\mathrm{n}}$ values

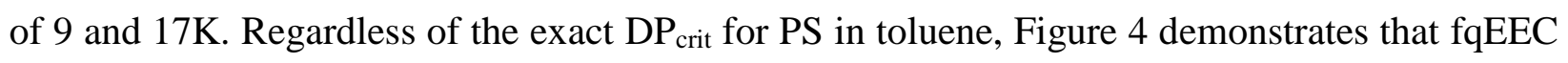
experiments conducted with pyrene end-labeled polymers become challenging for polymeric 
backbones that are stiffer than that of polystyrene such as polypeptides. Indeed if the LRPCD of such polymers were investigated by excimer formation between two pyrene end groups, the range of $N$ and $<k_{1}>$ values available for fqEEC would correspond to a rather small triangle in Figure 4. The $\mathrm{DP}_{\mathrm{on}}-\mathrm{DP}_{\text {crit }}$ range would span $N$ values between 40 and 340, a difference representing less than one order of magnitude variation in chain length. Furthermore an $N$ value of 40 at the lower end would correspond to a DP of 20 for vinyl polymers or 13 for peptides which would hardly qualify such samples as polymers. Based on the triangle drawn in Figure 4, the range of $<k_{1}>$ values would have lower and upper boundaries of $10^{6}$ and $10^{7} \mathrm{~s}^{-1}$, respectively, which also represents a rather short range to build a scaling law.

The use of longer-lived dyes such as phosphorescent tryptophan ${ }^{49}$ or anthracene ${ }^{66}$ would extend the range of $N$ and $<k_{1}>$ values accessible to fqEEC experiments. However it would also raise the possibility of having interpolymeric quenching events. Longer chains require larger massic polymer concentrations to maintain a dye concentration that is sufficiently high to ensure reasonable signal detection, particularly for long-lived luminophores having a relatively low molar extinction coefficient like DBO or weak luminescence quantum yield as for phosphorescent tryptophan. In summary, the procedure applied to build the $<k_{1}>\sim N^{-\gamma}$ trend is best suited to study oligomers and not polymers, above all if fluorescence is employed instead of other much longerlived photophysical processes. fqEEC experiments work well for polymers with a backbone that is more flexible than polystyrene such as poly(ethylene oxide) or polydimethylsiloxane as illustrated in Figure 4. For stiffer polymers such as polypeptides with large DPs comparable to those of proteins whose LRPCD would be most valuable to characterize, the above discussion leads to the conclusion that fqEEC experiments in their current form will struggle to deliver relevant information on their LRPCD since they will be limited to short oligopeptides making it 
difficult to generate the $\left\langle k_{1}>\propto N^{-\gamma}\right.$ relationship that enables one to compare the dynamics of different polymer backbones. Yet the overwhelming majority of experiments conducted to date with fluorescently labeled biological macromolecules has been carried out with end-labeled chains.

\section{PROBING LRPCD WITH RANDOMLY LABELED POLYMERS}

Despite the limitations of fqEEC experiments for the characterization of the LRPCD of stiff polymers in solution, quenching experiments conducted on fluorescently labeled macromolecules are still endowed with a number of worthwhile features, particularly their ability to probe isolated macromolecules in solution due to the outstanding sensitivity of fluorescence. This feature allows fluorescence experiments to be conducted at polymer concentrations that are so low that intermolecular interactions between fluorescently labeled macromolecules are prevented. As it turns out, not only can polymers randomly labeled with a dye and quencher be probed at concentrations that are lower than those used to study end-labeled polymers, but they also generate more dye-quencher encounters. As illustrated in Figure 5, a polystyrene sample randomly labeled with pyrene (PyBA-PS, chemical structure in Table 2) showed greatly enhanced pyrene excimer formation at $480 \mathrm{~nm}$ for a same pyrene content when compared to the pyrene end-labeled polystyrene equivalent (Pyz-PS(8K), see Table 2 for chemical structure). ${ }^{67,34}$

This massive enhancement in pyrene excimer formation can be explained as follows. Whereas two pyrene labels attached to the ends of a polymer have a $100 \%$ probability to be held far apart from each other, thus reducing dramatically the chance of EEC encounter for longer chains, the same two pyrene labels always have a non-zero probability to be attached close enough from each other along a chain of any length, and thus form excimer efficiently, as long as the polymer is randomly labeled with pyrene. Consequently if the purpose of a fluorescence quenching 
experiment is to probe the diffusive encounters between a dye and a quencher, Figure 5 indicates that randomly labeled chains are much better suited for this purpose than end-labeled chains.

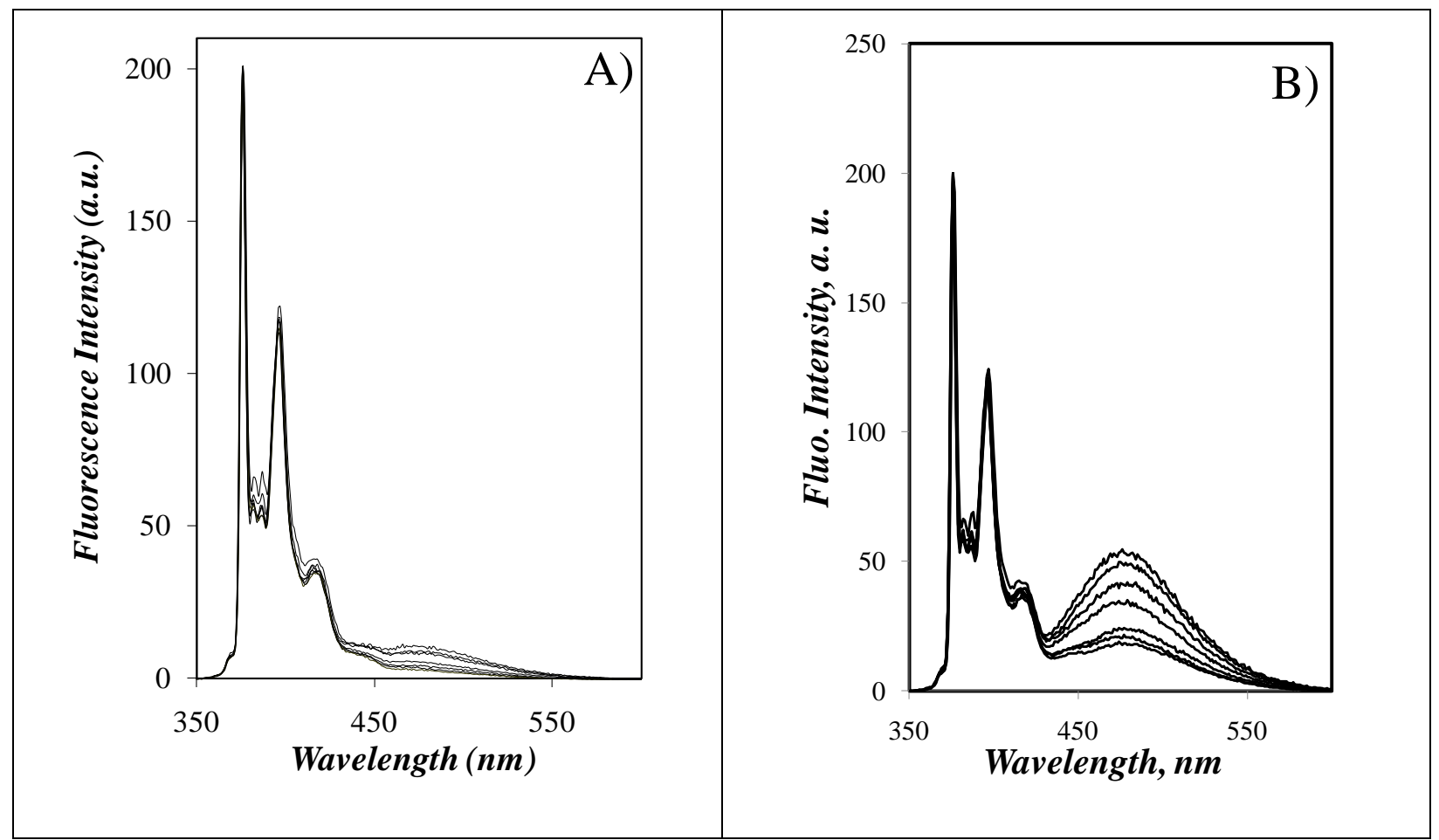

Figure 5. Steady-state fluorescence spectra of polystyrene A) Py2-PS(8K) with a 2.6 mol\% pyrene content ${ }^{34}$ and B) PyBA-PS with a 2.1 mol\% pyrene content. ${ }^{67}$ Solvents from top to bottom: methyl ethyl ketone, dichloromethane, tetrahydrofuran, toluene, dimethyl formamide, dioxane, and dimethyl acetamide. The chemical structure of the polymers is provided in Table 2.

Unfortunately the use of randomly labeled polymers to investigate their LRPCD in solution was complicated earlier on by the absence of a model such as Birks' scheme for pyrene end-labeled monodisperse polymers ${ }^{19}$ that would enable the analysis of the complex kinetics of quenching of an excited dye by quenchers randomly distributed along a polymer. The fluorescence blob model (FBM) was introduced in 1999 to fill this theoretical gap. ${ }^{35}$ The FBM defined the blob as the volume within the polymer coil probed by an excited dye. The blob could then be viewed as a unit 
volume that could be employed to compartmentalise the polymer coil into a cluster of blobs among which the quenchers would be randomly distributed according to a Poisson distribution. The equations that were derived by Tachiya ${ }^{71}$ to describe the quenching of dyes by quenchers randomly distributed among surfactant micelles could then be applied to the fluorescence decays of randomly labeled polymers by considering that dyes and quenchers randomly distributed among blobs would display the same quenching kinetics as if they were randomly distributed among surfactant micelles. To date, the FBM has been applied to the analysis of the monomer and excimer decays acquired with several polymers randomly labeled with pyrene. ${ }^{35,37,67,68,72-75}$ Through the FBM analysis of the fluorescence decays, the size of a blob, $N_{\text {blob }}$ expressed in terms of the number of monomers encompassed inside a $b l o b$, and the rate constant $k$ blob of excimer formation between one excited and one ground-state pyrene located inside a same blob could be determined. The pair of parameters $N_{\text {blob }}$ and $k$ blob obtained with randomly labeled polydisperse polymers showed remarkable similarity to the pair of parameters $N$ and $<k_{1}>$ obtained for the EEC of end-labeled monodisperse polymers. This equivalence was first demonstrated with pyrene-labeled polystyrenes (Py-PS).

Three series of Py-PS were prepared by copolymerizing styrene with 1pyrenemethylacrylamide, 1-pyrenebutyl acrylate, or 4-(1-pyrene)methoxymethylstyrene yielding PyAM-PS, PyBA-PS, and PyMe-PS, respectively, and their chemical structures are presented in Table 2. The fluorescence decays of the Py-PS constructs acquired in a wide range of solvents were analyzed according to the FBM recovering sets of $N_{\text {blob }}$ and $k_{\text {blob }} \times N_{\text {blob }}$ values that were averaged as a function of pyrene content to yield $<N_{\text {blob }}>$ and $<k_{\text {blob }} \times N_{\text {blob }}>$.

Each Py-PS construct yielded a different set of $<N_{\text {blob }}>$ and $<k_{\text {blob }} \times N_{\text {blob }}>$ parameters. This result underlined the importance of using a similar pyrene derivative for comparison purposes. Yet 
despite the different trends obtained for $\left\langle N_{\text {blob }}>\right.$ and $\left\langle k_{\text {blob }} \times N_{\text {blob }}>\right.$, these parameters could be normalized so that all $<N_{\text {blob }}>$ and $<k_{\text {blob }} \times N_{\text {blob }}>$ values fell on a master curve when plotted as a function of the inverse of solvent viscosity $\left(\eta^{-1}\right)$, an indication that these trends reported on the LRPCD of polystyrene in solution (Figure 6A) regardless of the chemical composition of the monomer bearing the pyrene label. ${ }^{67}$ Furthermore, the product $<k_{1}>\times N$ obtained for several pyrene end-labeled monodisperse polystyrenes yielded trends as a function of $\eta^{-1}$ that, after normalization, overlapped those obtained with $k_{\mathrm{blob}} \times N_{\text {blob }}$ for PyAM-PS, PyBA-PS, and PyMe-PS. ${ }^{20}$

Table 2. Chemical structure of the polystyrenes and poly( $N$-isopropylacrylamide) randomly labeled with pyrene.

PyMe-PS ${ }^{35,34}$

The overlapping trends obtained for the different Py-PS constructs demonstrated that they all reported in the same manner on the LRPCD of polystyrene, regardless of the mode of pyrene labeling selected to prepare the Py-PS constructs. Similar conclusions were reached one year later after comparing the products $k_{\text {blob }} \times N_{\text {blob }}$ and $<k_{1}>\times N$ for a series of poly( $N$-isopropylacrylamide) 
randomly and end-labeled with pyrene, respectively, where overlapping trends were obtained for both types of constructs. ${ }^{75}$ These trends are shown in Figure 6B. Together these studies demonstrated that short monodisperse end-labeled polymers with a DP between $\mathrm{DP}_{\mathrm{ON}}$ and $\mathrm{DP}_{\text {crit }}$ or long polydisperse polymers randomly labeled with pyrene yielded the same information on LRPCD. But instead of being limited to the study of oligomers as typically done with fqEEC experiments, FBM analysis could be applied to actual polymers with any DP as long as it was larger than that of a blob. In practice, that meant that, contrary to fqEEC experiments on endlabeled monodisperse oligomers, polymers with DPs much greater than 100 could be employed.

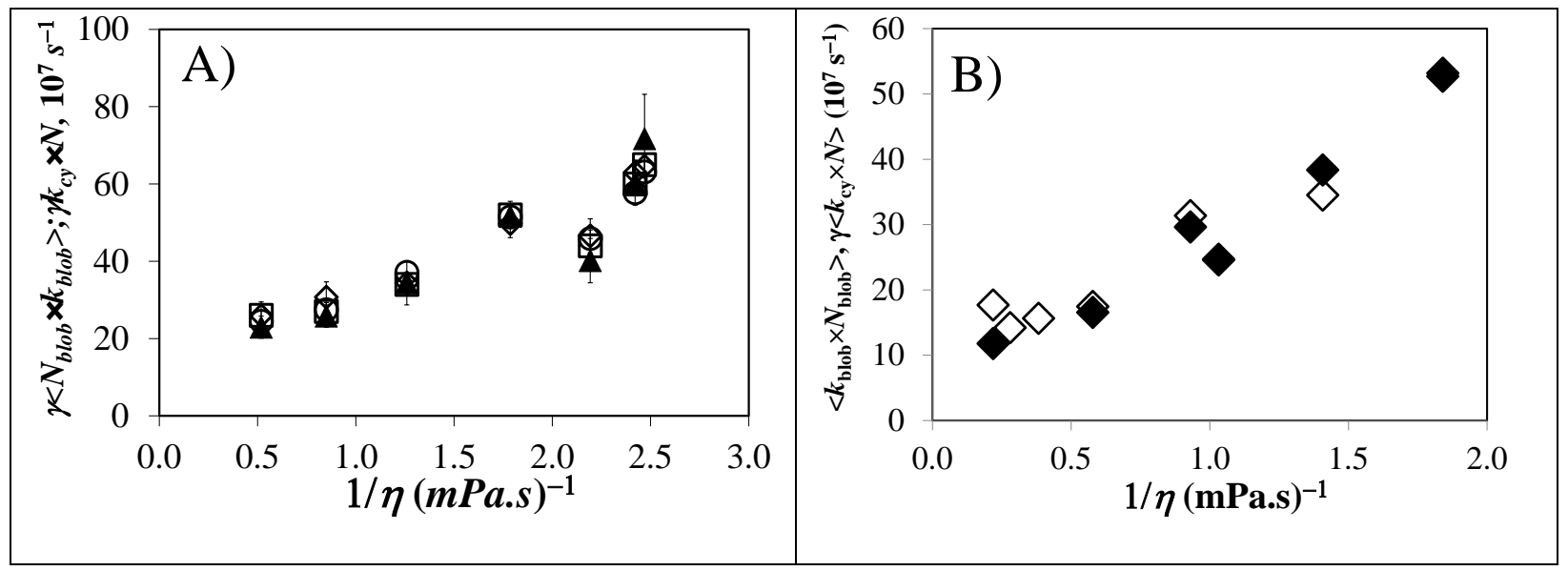

Figure 6. Plots of the products $<k_{\text {blob }} \times N_{\text {blob }}>$ and $<k_{1}>\times N$ after normalization as a function of the inverse of solvent viscosity for A) Py2-PS ( $\mathbf{\Delta})$, PyAM-PS ( $\square$ ), PyBA-PS (O), PyMe-PS $(\diamond)$ and B) PyBAM-PNIPAM $(\diamond)$, Py2$_{2}-\operatorname{PNIPAM}(\diamond)$.

These studies demonstrated that the product $<k_{\mathrm{blob}} \times N_{\mathrm{blob}}>$ for randomly labeled polymers contained the same dynamic information as the product $<k_{1}>\times N$ for end-labeled monodisperse oligomers. However, they did not demonstrate that $<k_{\text {blob }} \times N_{\text {blob }}>$ provides a faithful representation of LRPCD. To do so, $<k_{b l o b} \times N_{b l o b}>$ needed to be determined and compared for polymers whose 
chemical composition was known to result in different LRPCD. How this was accomplished is described hereafter.

\section{PRODUCT $<k_{b l o b} \times N_{\text {blob }}>$ TO DESCRIBE LRPCD IN SOLUTION}

The ability to work with actual polymers when applying the FBM led to the study of the effect that the chemical structure of the side chain of poly(alkyl methacrylate)s would have on their LRPCD in tetrahydrofuran (THF). Poly(alkyl methacrylate)s randomly labeled with pyrene were prepared through copolymerization of 1-pyrenebutyl methacrylate with either methyl methacrylate, $n$-butyl methacrylate, $n$-hexyl methacrylate, $n$-octyl methacrylate, $n$-dodecyl methacrylate, $n$-stearyl methacrylate, tert-butyl methacrylate, or cyclohexyl methacrylate to yield Py-PC1MA, PyPC4MA, Py-PC6MA, Py-PC8MA, Py-PC12MA, Py-PC18MA, Py-PC4TBMA, or Py-PC6CyMA, respectively. ${ }^{68}$ The chemical structures of these pyrene-labeled poly(alkyl methacrylate)s are presented in Table 3. The longer side chains of Py-PC12MA and Py-PC18MA were expected to slow down the LRPCD of these poly(alkyl methacrylate)s to the point that no excimer would be generated if the chains had been end-labeled for fqEEC experiments (see Figure 4). For each polymer series, five pyrene labeled polymers were prepared with pyrene content ranging between 2 and $10 \mathrm{~mol} \%$ and their monomer and excimer decays were acquired.

The global FBM analysis of the monomer and excimer fluorescence decays yielded the parameters $N_{\text {blob }}$ and the product $k_{\text {blob }} \times N_{\text {blob. }}$. Within experimental error, they were found to remain constant with pyrene content. They were averaged and $<N_{\text {blob }}>$ and $<k_{\text {blob }} \times N_{\text {blob }}>$ were plotted as a function of side chain length in Figure 7A and B, respectively. $<N_{\text {blob }}>$ decreased with increasing side chain length reflecting the smaller blob probed by an excited pyrene when the LRPCD of the poly(alkyl methacrylate)s were slowed down by the bulkier side chains. A similar trend was 
observed for $<k_{\text {blob }} \times N_{\text {blob }}>$. For the longer side chains, $<k_{\text {blob }} \times N_{\text {blob }}>$ remained constant indicating that side chain length no longer had an effect on LRPCD for side chains longer than $n$-dodecyl.

Table 3. Chemical structure of the poly(alklyl methacrylate)s randomly labeled with pyrene

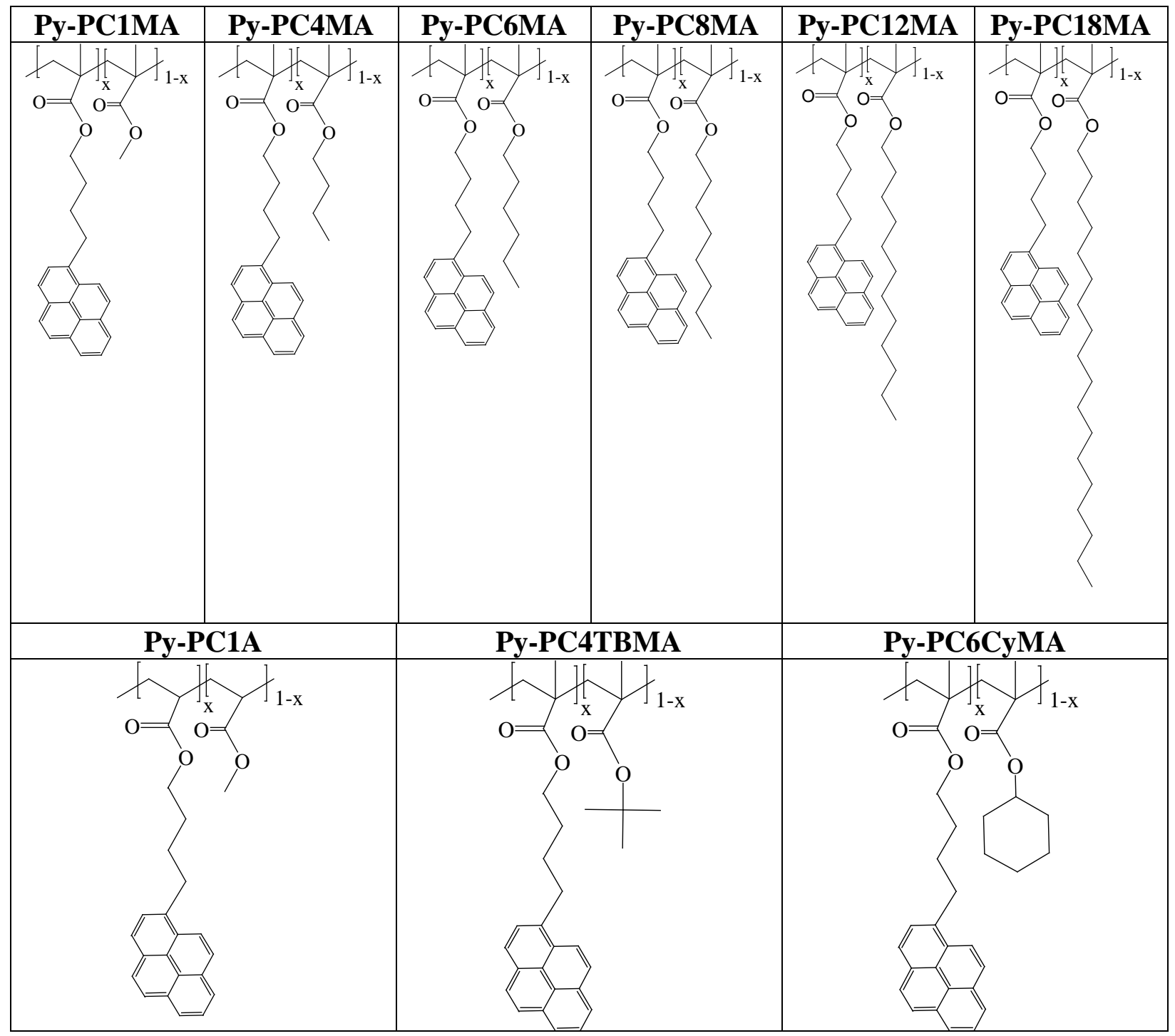

The effect of the nature of the side chain on LRPCD in solution was also investigated by comparing $<N_{\text {blob }}>$ and $<k_{\text {blob }} \times N_{\text {blob }}>$ obtained for Py-PC4MA and Py-PC4TBMA on the one hand and Py-PC6MA and Py-PC6CyMA on the other hand (see corresponding structures in Table 3). Both $<N_{\text {blob }}>$ and $<k_{\text {blob }} \times N_{\text {blob }}>$ were reduced upon changing the side chain from $n$-butyl in Py- 
PC4MA to tert-butyl in Py-PC4TBMA or from n-hexyl in Py-PC6MA to cyclohexyl in PyPC6CyMA. These results agreed with expectations that LRPCD should be reduced upon rigidifying the side chain of poly(alkyl methacrylate)s. Surprisingly, $<N_{\mathrm{blob}}>$ was found to be the same for Py-PC1MA and Py-PC1A despite the fact that PC1A should have been much more flexible than PC1MA, and thus should have allowed an excited pyrene to probe a larger blob resulting in a larger $N_{\text {blob }}$ value. The reason for this apparent inconsistency was rooted in the fact that $N_{\text {blob }}$ is a measure of the size of the blob, not the frequency at which the blob volume is being probed by an excited pyrene. The parameter that describes this frequency, and thus better represents LRPCD, is the product $<k_{\text {blob }} \times N_{\text {blob }}>$. As expected, $<k_{\text {blob }} \times N_{\text {blob }}>$ at $0.82 \mathrm{~ns}^{-1}$ was twice larger for Py-PC1A than for Py-PC1MA for which $<k_{\text {blob }} \times N_{\text {blob }}>$ equalled $0.41 \mathrm{~ns}^{-1}$.

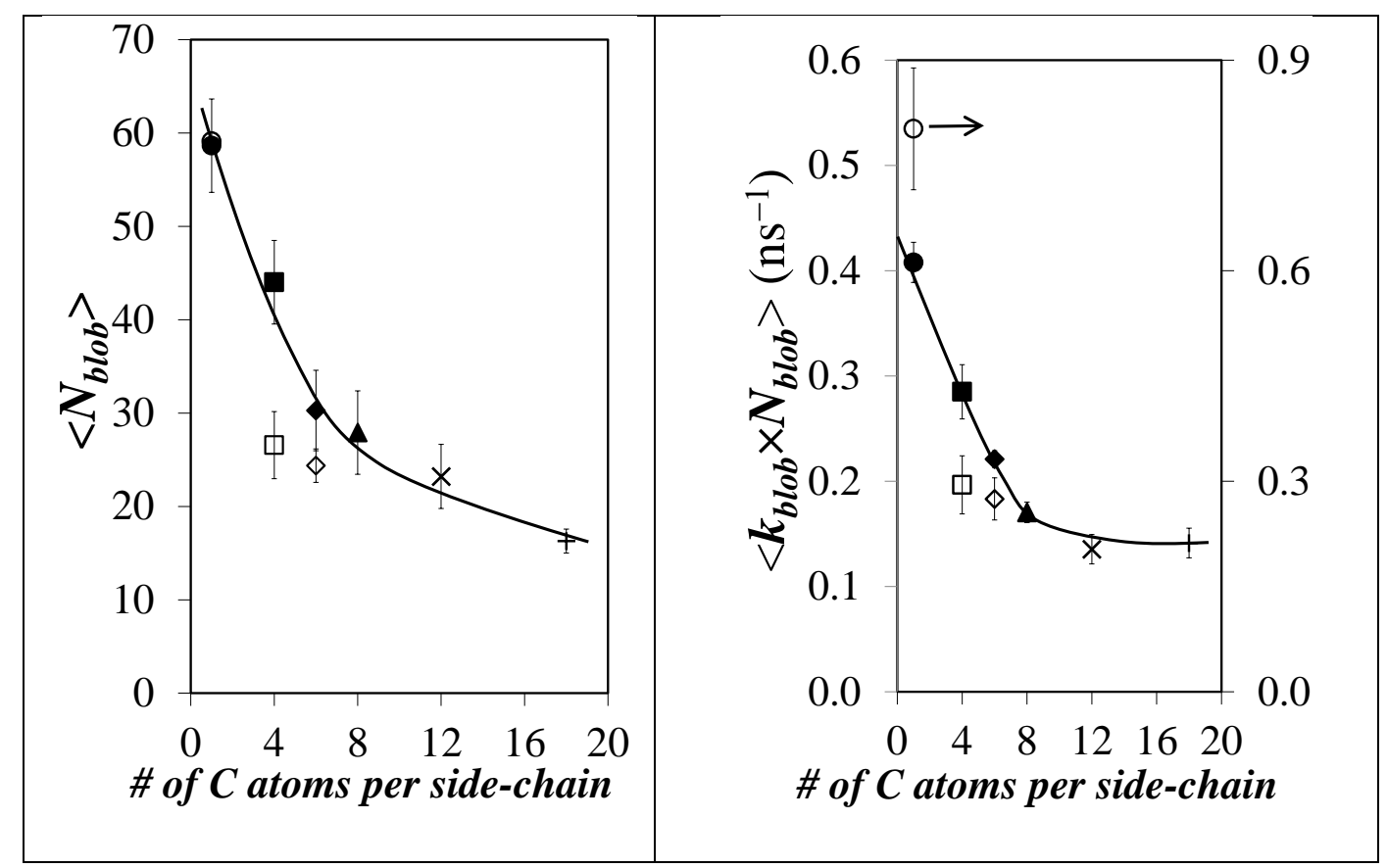

Figure 7. Plot of A) $<N_{\text {blob }}>$ and B) $<k_{\text {blob }} \times N_{\text {blob }}>$ as a function of the number of carbon atoms per side chain. Left axis: $($ ) Py-PC1MA, ( $\boldsymbol{\square})$ Py-PC4MA, $(\boldsymbol{\square})$ Py-PC4TMA, $(\diamond)$ Py-PC6MA, $(\diamond)$ Py-PC6CyMA, ( $\mathbf{\Delta}$ ) Py-PC8MMA, (×) Py-PC12MA, (+) Py-PC18MA. Right axis: (O) Py-PC1A. 
The trends shown in Figure $7 \mathrm{~B}$ for $<k_{\mathrm{blob}} \times N_{\mathrm{blob}}>$ are in agreement with their expected LRPCD predicted from their $T_{\mathrm{g}}$ values in the solid state which suggests that $<\mathrm{kblob}_{\mathrm{b}} \times N_{\mathrm{blob}}>$ could be employed as a universal parameter to describe the LRPCD in solution. Most importantly, $<k_{\text {blob }} \times N_{\text {blob }}>$ for polystyrene was found to equal $0.53 \mathrm{~ns}^{-1}$ indicating that PS is less flexible than PC1A but much more flexible than any of the poly(alkyl methacrylate)s listed in Table 3. Indeed, the most flexible poly(alkyl methacrylate), namely PC1MA, had a $<k_{\mathrm{blob}} \times N_{\mathrm{blob}}>$ value of $0.41 \mathrm{~ns}^{-1}$. Since polystyrene represents one of the stiffest polymeric backbones whose LRPCD can be probed by fqEEC experiments using pyrene excimer fluorescence (see Figure 4), it is worth pointing out at this stage that the LRPCD of none of the poly(alkyl methacrylate)s listed in Table 3 could have been determined by conducting a fqEEC study. Last but not least, the comparison of the $<k_{1}>$ values in Figure 4 for three polymer backbones was the summary of four different studies. By contrast, the comparison of the $<k_{\mathrm{blob}} \times N_{\mathrm{blob}}>$ values in Figure 7 for nine different polymer backbones was conducted in a single study. The ease of dealing with polydisperse randomly labeled polymers makes it considerably simpler to study LRPCD when compared to what can be accomplished with end-labeled monodisperse polymers that are usually much more challenging to prepare.

\section{RELEVANCE OF FLUORESCENCE QUENCHING EXPERIMENTS TO STUDY PROTEIN FOLDING}

As this review has illustrated, fluorescence quenching experiments on linear chains have been instrumental in providing robust experimental tools to probe the LRPCD of synthetic polymers and resulted in trends that are general for any linear chain, and thus must also be obeyed by biological polymers such as polypeptides. ${ }^{28,30,43-62}$ Fluorescence quenching EEC experiments carried out on end-labeled oligopeptides provide a direct measure of the time scale over which the 
most elementary step in protein folding takes place, namely loop formation. These experiments, together with FBM studies on randomly labeled polymers, have enabled the comparison of the LRPCD of different polymeric backbones. But another possibly important impact of fluorescence quenching experiments might be the demonstration through the FBM analysis of fluorescence decays of randomly labeled polymers that the mobility of a monomer in a chain is limited to a subvolume of the polymer coil. As pointed out in a 2006 article, ${ }^{37}$ this insight could help resolve Levinthal's famous paradox. ${ }^{76}$

In 1969, Levinthal pointed out that a protein made of a large number of amino acids would take an infinitely long time to sample its entire conformational space before folding into its final 3-dimensional structure. ${ }^{38}$ Indeed, if the protein was made of 300 amino acids (aa), each taking 1 ps to probe three possible conformations, it would take $3^{300} \times 1$ ps $=4.3 \times 10^{121}$ centuries for the 300 aa-long protein to fold. Since proteins are capable of folding within the much shorter lifetime span of living organisms, Levinthal's paradox demonstrated that proteins could not probe the entire conformational space. This conclusion led scientists to design theories that would enable a protein to fold without having to probe its entire conformational space. Such theories include the framework model, ${ }^{77}$ the nucleation model, ${ }^{78,79}$ the hydrophobic-collapse model, ${ }^{80}$ or a folding pathway along an energy funnel, ${ }^{81,82}$ to name but a few. Yet, if the theoretical framework of the FBM is correct and the monomers of a chain can only probe a subvolume of a polymer coil called a $b l o b$, then the folding time of a protein would be that required for a blob to fold times the number

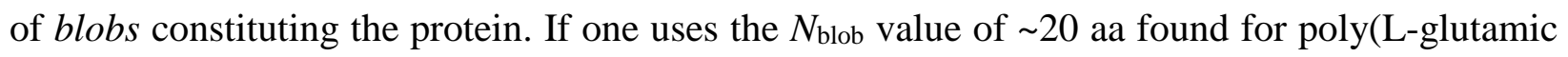
acid) labeled with 1-pyrenemethylamine and studied with the $\mathrm{FBM},{ }^{73,83}$ the same 300 aa protein discussed earlier would be made of 15 blobs and its folding time would decrease from $4.3 \times 10^{121}$ centuries down to $15 \times 3^{20} \times 1 \mathrm{ps}=52 \mathrm{~ms}$, a much more reasonable folding time. As a matter of fact, 
the blobs determined by the FBM might be equivalent to the recently introduced foldons, a foldon being a $\sim 20$ aa segment of a protein where folding is initially believed to occur. ${ }^{11}$ The foldons might very well be equivalent to the blobs introduced as early as 1993 in an early coarse version of the FBM as the loci where folding of a much longer chain is believed to take place. ${ }^{84}$

\section{CONCLUSIONS}

This Perspective has provided a summary of the different types of fluorescence quenching experiments that have been conducted to date to characterize the LRPCD of linear chains. Such studies can be divided into fqEEC experiments where a linear chain is end-labeled with a dye and a quencher and FBM experiments on linear chains randomly labeled with pyrene. While fqEEC experiments have demonstrated their potential to probe LRPCD, several drawbacks of the procedure have been highlighted that impede its ready application to study the LRPCD of a broad range of polymeric backbones, in particular for long polymers with a rigid backbone. By contrast, FBM experiments can be applied to polydisperse polymers having a large degree of polymerization and a rigid backbone.

Regardless of the different merits of conducting fluorescence quenching experiments on end- or randomly labeled linear chains, this Perspective has also demonstrated that fluorescence quenching experiments represent a powerful and robust analytical tool to obtain reliable information on the LRPCD of linear chains, the time scale of the most elementary step taking place in protein folding (i.e. loop formation), and the size of the subdomain of a protein where folding of a protein actually takes place (i.e. a foldon). These achievements to date pave the way to future fruitful and numerous discoveries in the study of the internal dynamics of macromolecules in solution based on fluorescence quenching experiments. 


\section{REFERENCES}

1. Glass, J. E., Ed.; Polymers in Aqueous Media: Performance through Association; Advances in Chemistry Series 226; American Chemical Society: Washington, DC, 1989.

2. Schulze, D. N.; Glass, J. E., Ed.; Polymers as Rheology Modifiers; ACS Symposium Series 462; American Chemical Society: Washington, DC, 1991.

3. Kwak, J. C. T., Ed.; Polymer-Surfactant Systems; Surfactant Science Series 77; Marcel Dekker: New York, 1998.

4. Goddard, E. O.; Ananthapadamanabham, K. P., Ed.; Interactions of Surfactants with Polymers and Proteins; CRC Press: Boca Raton, FL, 1993.

5. Winnik, M. A.; Yekta, A. Associative Polymers in Aqueous Solution. Curr. Opin. Colloid Interface Sci. 1997, 2, 424-436.

6. Glass, J. E. A Perspective on the History of and Current Research in Surfactant-Modified, Water-Soluble Polymers. J. Coat. Tech. 2001, 73, 79-98.

7. Bai, Y. Protein Folding Pathways Studied by Pulsed- and Native-State Hydrogen Exchange. Chem. Rev. 2006, 106, 1757-1768.

8. Neira, J. L. NMR as a Tool to Identify and Characterize Protein Folding Intermediates. Archiv. Biochemistry Biophys. 2013, 531, 90-99.

9. Adhikari, A. N.; Freed, K. F.; Sosnick, T. T. Simplified Protein Models: Predicting Folding Pathways and Structure Using Amino Acid Sequences. Phys. Rev. Lett. 2013, 111, 028103.

10. Plotkin, S. S.; Onuchic, J. N. Understanding Protein Folding with Energy Landscape Theory. Part I: Basic Concepts. Q. Rev. Biophys. 2002, 35, 111-167. 
11. Englander, S. W.; Mayne, L. The Nature of Protein Folding Pathways. Proc. Natl. Acad. Sci. 2014, 111, 15873-15880.

12. Li, J.; Ngai, T.; Wu, C. The Slow Relaxation Mode: From Solutions to Gel Networks. Polym. J. 2010, 42, 609-625.

13. Dais, P.; Spyros, A. 13C Nuclear Magnetic Relaxation and Local Dynamics of Synthetic Polymers in Dilute Solution and in the Bulk State. Prog. Nucl. Magn. Res. Spectrosc. 1995, 27, 555-633.

14. Pilař, J. Local Dynamics of Polymers in Solution by Spin-Label ESR. In Advance ESR Methods in Polymer Research, Ed. Schlick, S. Wiley, 2006, pp 133-163.

15. Hyun, K.; Wilhelm, M.; Klein, C. O.; Cho, K. S.; Nam, J. G.; Ahn, K. H.; Lee, S. J.; Ewoldt, R. H.; McKinley, G. H. A Review of Nonlinear Oscillatory Shear Tests: Analysis and Application of Large Amplitude Oscillatory Shear (LAOS). Porg. Polym. Sci. 2011, 36, 16971753.

16. Perkins, T. T.; Quake, S. R.; Smith, D. E.; Chu, S. Relaxation of a Single DNA Molecule Observed by Optical Microscopy. Science 1994, 264, 822-826.

17. Keshavarz, M.; Engelkamp, H.; Xu, J.; Braeken, E.; Otten, M. B. J.; Uji-i, H.; Schwartz, E.; Koepf, M.; Vananroye, A.; Vermant, J.; Nolte, R. J. M.; De Schryver, F.; Maan, J. C.; Hofkens, J.; Christianen, P. C. M., Rowan, A. E. Nanoscale Study of Polymer Dynamics. ACSNano 2016, 10, 1434-1441.

18. Viovy, J. L.; Monerie, L. Fluorescence Anisotropy Technique Using Synchrotron Radiation as a Powerful Means for Studying the Orientation Correlation Function of Polymer Chains. Adv. Polym. Sci. 1985, 67, 99-122.

19. Winnik, M. A. End-to-End Cyclization of Polymer Chains. Acc. Chem. Res. 1985, 18, 73-79. 
20. Duhamel, J. New Insights in the Study of Pyrene Excimer Fluorescence to Characterize Macromolecules and their Supramolecular Assemblies in Solution. Langmuir 2012, 28, 65276538.

21. Duhamel, J. Global Analysis of Fluorescence Decays to Probe the Internal Dynamics of Fluorescently Labeled Macromolecules. Langmuir 2014, 30, 2307-2324.

22. Mayor, U.; Guydosh, N. R.; Johnson, C. M.; Grossmann, J. G.; Sato, S.; Jas, G. S.; Freund, S. M. V.; Alonse, D. O. V.; Daggett, V.; Fersht, A. R. The Folding Pathway of a Protein from Nanoseconds to Microseconds. Nature 2003, 421, 863-867.

23. Stryer, L.; Haugland, R. P. Energy Transfer: A Spectroscopic Ruler. Proc. Natl. Acad. Sci. 1967, 58, 719-726.

24. Haas, E.; Katchalski-Katzir, E.; Steinberg, I. Z. Brownian Motion of the Ends of Oligopeptide Chains in Solution as Estimated by Energy Transfer between the Chain Ends. Biopolymers 1978, $17,11-31$.

25. Winnik, M. A.; Redpath, T.; Richards, D. H. The Dynamics of End-to-End Cyclization in Polystyrene Probed by Pyrene Excimer Formation. Macromolecules 1980, 13, 328-335.

26. Huang, F.; Hudgins, R. R.; Nau, W. M. Primary and Secondary Structure Dependence of Peptide Flexibility Assessed by Fluorescence-Based Measurement of End-to-End Collision Rates. J. Am. Chem. Soc. 2004, 126, 16665-16675.

27. Möglich, A.; Joder, K.; Kiefhaber, T. End-to-End Distance Distributions and Intrachain Diffusion Constants in Unfolded Polypeptide Chains Indicate Intramolecular Hydrogen Bond Formation. Proc. Natl. Acad. Sci. 2006, 103, 12394-12399.

28. Brucale, M.; Schuler, B.; Samori, B. Single-Molecule Studies of Intrinsically Disordered Proteins. Chem. Rev. 2014, 114, 3281-3317. 
29. Duhamel, J.; Winnik, M. A.; Baros, F.; André, J.-C.; Martinho, J. M. G. Diffusion Effects on Pyrene Excimer Kinetics: Determination of the Excimer Formation Rate Coefficient Time Dependence. J. Phys. Chem. 1992, 96, 9805-10.

30. Sustarsic, M.; Kapanidis, A. N. Taking the Ruler to the Jungle: Single-Molecule FRET for Understanding Biomolecular Structure and Dynamics in Living Cells. Curr. Opin. Struct. Biol. 2015, 34, 52-59.

31. Bains, G. K.; Kim, S. H.; Sorin, E. J.; Narayanaswami, V. The Extent of Pyrene Excimer Fluorescence Emission is a Reflector of Distance and Flexibility: Analysis of the Segment Linking the LDL Receptor-Binding and Tetramerization Domains of Apolipoprotein E3. Biochemistry 2012, 51, 6207-6219.

32. Mizuguchi, C.; Hata, M.; Dhanasekaran, P.; Nickel, M.; Phillips, M. C.; Lund-Katz, S.; Saito, H. Fluorescence Analysis of the Lipid Binding-Induced Conformational Change of Apolipoprotein E4. Biochemistry 2012, 51, 5580-5588.

33. König, I.; Zarrine-Afsar, A.; Aznauryan, M.; Soranno, A.; Wunderlich, B.; Dingfelder, F.; Stüber, J. C.; Plückthun, A.; Nettels, D.; Schuler, B. Single-Molecule Spectroscopy of Protein Conformational Dynamics in Live Eukaryotic Cells. Nature Meth. 2015, 12, 773-779.

34. Ingratta, M.; Hollinger, J.; Duhamel, J. A Case for Using Randomly Labeled Polymers to Study Long Range Polymer Chain Dynamics by Fluorescence. J. Am. Chem. Soc. 2008, 130, 9420-9428.

35. Mathew, A.; Siu, H.; Duhamel, J. A Blob Model to Study Chain Folding by Fluorescence. Macromolecules 1999, 32, 7100-7108.

36. Sperling, L. H. Physical Polymer Science, $4^{\text {th }}$ Ed. Wiley, Bethlehem, 2006, pp 375-376. 
37. Irondi, K.; Zhang, M.; Duhamel, J. Study of the Semidilute Solutions of Poly $(N, N-$ dimethylacrylamide) by Fluorescence and its Implications to the Kinetics of Coil-to-Globule Transition. J. Phys. Chem. B 2006, 110, 2628-2637.

38. Levinthal, C. How to Fold Graciously. Mossbauer Spectroscopy in Biological Systems. Proceedings University of Illinois Bulletin (University of Illinois Press, Urbana, IL), 1969, pp $22-24$.

39. Birks, J. B.; Dyson, D. J.; Munro, I. H. ‘Excimer' Fluorescence. II. Lifetime Studies of Pyrene Solutions Proc. Roy. Soc. A 1963, 275, 575-588.

40. Ghiggino, K. P.; Snare, M. J.; Thistlethwaite, P. J. Cyclization Dynamics in Poly(ethylene oxide). Chain Length and Temperature Dependence. Eur. Polym. J. 1985, 21, 265-272.

41. Svirskaya, P.; Danhelka, J.; Redpath, A. E. C.; Winnik, M. A. Cyclization Dynamics of Polymers: 7. Applications of the Pyrene Excimer Technique to the Internal Dynamics of Polydimethylsiloxane Chains. Polymer 1983, 24, 319-322.

42. Boileau, S.; Mechin, F.; Martinho, J. M.; Winnik, M. A. End-to-End Cyclization of a Pyrene End-Capped Poly(bisphenol A-diethylene glycol carbonate). Macromolecules 1989, 22, 215220.

43. Fierz, B.; Kiefhaber, T. End-to-End vs Interior Loop Formation Kinetics in Unfolded Polypeptide Chains. J. Am. Chem. Soc. 2007, 129, 672-679.

44. Fierz, B.; Satzger, H.; Root, C.; Gilch, P.; Zinth, W.; Kiefhaber, T. Loop Formation in Unfolded Polypeptide Chains on the Picoseconds to Microseconds Time Scale. Proc. Natl. Acad. Sci. 2007, 104, 2163-2168. 
45. Hagen, S. J.; Hofrichter, J.; Szabo, A.; Eaton, W. A. Diffusion-Limited Contact Formation in Unfolded Cytochrome c: Estimating the Maximum Rate of Protein Folding. Proc. Natl. Acad. Sci. 1996, 93, 11615-11617.

46. Hudgins, R. R.; Huang, F.; Gramlich, G.; Nau, W. M. A Fluorescence-Based Method for Direct Measurement of Submicrosecond Intramolecular Contact Formation in Biopolymers: An Exploratory Study with Polypeptides. J. Am. Chem. Soc. 2002, 124, 556-564.

47. Huang, F.; Nau, W. M. A Conformational Flexibility Scale for Amino Acids in Peptides. Angew. Chem. Int. Ed. 2003, 42, 2269-2272.

48. Roccatano, D.; Sahoo, H.; Zacharias, M. ; Nau, W. M. Temperature Dependence of Looping Rates in a Short Peptide. J. Phys. Chem. B 2007, 111, 2639-2646.

49. Lapidus, L. S.; Eaton, W. A.; Hofrichter, J. Measuring the Rate of Intramolecular Contact Formation in Polypeptides. Proc. Natl. Acad. Sci. 2000, 97, 7220-7225.

50. Lapidus, L. J.; Eaton, W. A.; Hofrichter, J. Measuring Dynamic Flexibility of the Coil State of a Helix-Forming Peptide. J. Mol. Biol. 2002, 319, 19-25.

51. Haas, E. The Study of Protein Folding and Dynamics by Determination of Intramolecular Distance Distributions and Their Fluctuations Using Ensemble and Single-Molecule FRET Measurements. ChemPhysChem 2005, 6, 858-870.

52. Bieri, O.; Wirz, J.; Hellrung, B.; Schutkowski, M.; Drewello, M.; Kiefhaber, T. The Speed Limit for Protein Folding Measured by Triplet-Triplet Energy Transfer. Proc. Natl. Acad. Sci. 1999, 96, 9597-9601.

53. Krieger, F.; Fierz, B.; Bieri, O.; Drewello, M.; Kiefhaber, T. Dynamics of Unfolded Polypeptide Chains as Model for the Earliest Steps in Protein Folding. J. Mol. Biol. 2003, 332, 265-274. 
54. Möglich, A.; Krieger, F.; Kiefhaber, T. Molecular Basis for the Effect of Urea and Gaunidinium Chloride on the Dynamics of Unfolded Polypeptide Chains. J. Mol. Biol. 2005, 345, 153-162.

55. Fierz, B.; Kiefhaber, T. End-to-End vs Interior Loop Formation Kinetics in Unfolded Polypeptide Chains. J. Am. Chem. Soc. 2007, 129, 672-679.

56. Neuweiler, H.; Schultz, A.; Böhmer, M.; Enderlein, J.; Sauer, M. Measurements of Submicrosecond Intramolecular Contact Formation in Peptides at the Single-Molecule Level. J. Am. Chem. Soc. 2007, 125, 5324-5330.

57. McGimpsey, W. G.; Chen, L.; Carraway, R.; Samaniego, W. N. Singlet-Singlet and TripletTriplet Energy Transfer in Biochromophoric Peptides. J. Phys. Chem. A 1999, 103, 60826090.

58. Sahoo, H.; Roccatano, D.; Hennig, A.; Nau, W. M. A 10-ÅSpectroscopic Ruler Applied to Short Polyprolines. J. Am. Chem. Soc. 2007, 129, 9762-9772.

59. Jacob, M. H.; Dsouza, R. N.; Ghosh, I.; Norouzy, A.; Schwarzlose, T.; Nau, W. M. DiffusionEnhanced Förster Resonance Energy Transfer and the Effects of External Quenchers and the Donor Quantum Yield. J. Phys. Chem. B 2013, 117, 185-198.

60. Norouzy, A.; Assaf, K. I.; Zhang, S.; Jacob, M. H.; Nau, W. M. Coulomb Repulsion in Short Polypeptides. J. Phys. Chem. B 2015, 119, 33-43.

61. Galoppini, E.; Fox, M. A. Effect of the Electric Field Generated by the Helix Dipole on Photoinduced Intramolecular Electron Transfer in Dichromophoric $\alpha$-Helical Peptides. J. Am. Chem. Soc. 1996, 118, 2299-2300.

62. Fox. M. A.; Galoppini, E. Electric Field Effects on Electron Transfer Rates in Dichromophoric Peptides: The Effect of Helix Unfolding. J. Am. Chem. Soc. 1997, 119, 5277-5285. 
63. Schuler, B.; Soranno, A.; Hofmann, H.; Nettels, D. Single-Molecule FRET Spectroscopy and the Polymer Physics of Unfolded and Intrinsically Disordered Proteins. Ann. Rev. Biophys. 2016, 45, 207-231.

64. Sorano, A.; Koenig, I.; Borgia, M. B.; Hofmann, H.; Zosel, F.; Nettels, D.; Schuler, B. SingleMolecule Spectroscopy Reveals Polymer Effects of Disordered Proteins in Crowded Environments. Proc. Natl. Acad. Sci. 2014, 111, 4874-4879.

65. Schuler, B.; Lipman, E. A.; Steinbach, P. J.; Kumke, M.; Eaton, W. A. Polyproline and the “Spectroscopic Ruler” Revisited with Single-Molecule Fluorescence. Proc. Natl. Acad. Sci. 2005, 102, 2754-2759.

66. Horie, K.; Schnabel, W.; Mita, I.; Ushiki, H. Rates of Intramolecular Collision between Terminal Groups of $\alpha, \omega$-Dianthrylpolystyrene in Benzene and Cyclohexane Solutions as Studied by Triplet-Triplet Absorptiono Measurements. Macromolecules 1981, 14, 1422-1428.

67. Ingratta, M.; Mathew, M.; Duhamel,J. How Switching the Substituent of a Pyrene Derivative from a Methyl to a Butyl Affects the Fluorescence Response of Polystyrene Randomly Labeled with Pyrene. Can. J. Chem. 2010, 88, 217-227.

68. Farhangi, S.; Weiss, H.; Duhamel, J. Effect of Side-Chain Length on the Polymer Chain Dynamics of Poly (alkyl methacrylate) s in Solution. Macromolecules 2013, 46, 9738-9747.

69. Chen, S.; Duhamel, J.; Winnik, M. A. Probing End-to-End Cyclization Beyond Willemski and Fixmann. J. Phys. Chem. B 2011, 115, 3289-3302.

70. Brandrup, J. ; Immergut, E. H.; Grulke, E. A. Polymer Handbook, 4th ed.; John Wiley \& Sons: NY, 1999, p VII 675-683. 
71. Tachiya, M. Stochastic and Diffusion Models of Reactions in Micelles and Vesicles. In Kinetics of Nonhomogeneous Processes: A Practical Introduction for Chemists, Biologists, Physicists, and Material Scientists. Ed. G. R. Freeman, Wiley, NY, 1987, pp575-650.

72. Kanagalingam, S.; Spartalis, J.; Cao, T.-C.; Duhamel, J. Scaling Relations Related to the Kinetics of Excimer Formation between Pyrene Groups Attached onto Poly(N,Ndimethylacrylamide)s Macromolecules 2002, 35, 8571-8577.

73. Duhamel, J.; Kanagalingam, S.; O’Brien, T.; Ingratta, M. Side-Chain Dynamics of an $\alpha-$ Helical Polypeptide Monitored by Fluorescence. J. Am. Chem. Soc. 2003, 125, 12810-12822.

74. Teertstra, S. J.; Lin, W. Y.; Gauthier, M.; Ingratta, M. Duhamel, J. Comparison of the Long Range Polymer Chain Dynamics of Polystyrene and cis-Polyisoprene Using Polymers Randomly Labeled with Pyrene. Polymer 2009, 50, 5456-5466.

75. Yip, J.; Duhamel, J.; Qiu, X. P.; Winnik, F. M. Long-Range Polymer Chain Dynamics of Pyrene-Labeled Poly( $N$-isopropylacrylamide)s Studied by Fluorescence. Macromolecules 2011, 44, 5363-5372.

76. Levinthal, C. How to Fold Graciously. Mossbauer Spectroscopy in Biological Systems. Proceedings University of Illinois Bulletin (University of Illinois Press, Urbana, IL), 1969, pp $22-24$.

77. Ptitsyn, O. B. Kinetic and Equilibrium Intermediates in Protein Folding. Protein Eng. 1994, 7, 593-596.

78. Wetlaufer, D. B. Nucleation, Rapid Folding, and Globular Intrachain Regions in Proteins. Proc. Natl. Acad. Sci. U.S.A. 1973, 70, 697-701.

79. Wetlaufer, D. B. Nucleation in Protein Folding-Confusion of Structure and Process. Trends Biochem. Sci. 1990, 15, 414-415. 
80. Dill, K. A.; Bromberg, S.; Yue, K.; Fiebig, K. M.; Yee, D. P.; Thomas, P. D.; Chan, H. S. Principle of Protein Folding-A Perspective from Simple Exact Model. Protein Sci. 1995, 4, 561-602.

81. Dill, K. A.; Chan, H. S. From Levinthal to Pathways to Funnels. Nature Struct. Biol. 1997, 4, 10-19.

82. Dinner, A. R.; Sÿali, A.; Smith, L. J.; Dobson, C. M.; Karplus, M. Understanding Protein Folding via Free-Energy Surfaces from Theory to Experiment. Trends Biochem. Sci. 2000, 25, 331-339

83. Ingratta, M.; Duhamel, J. Effect of Side-chain Length on the Side-chain Dynamics of a-Helical Poly(L-glutamic acid) as Probed by a Fluorescence Blob Model. J. Phys. Chem. B 2008, 112, 9209-9218.

84. Duhamel, J.; Yekta, A.; Winnik, M. A.; Jao, T.-C.; Mishra, M. K.; Rubin, I. D. A Statistical Blob Model for Studying Polymer Chain Dynamics in Solution by Excimer Formation Processes. J. Phys. Chem. 1993, 97, 13708-13712. 
Table of Content

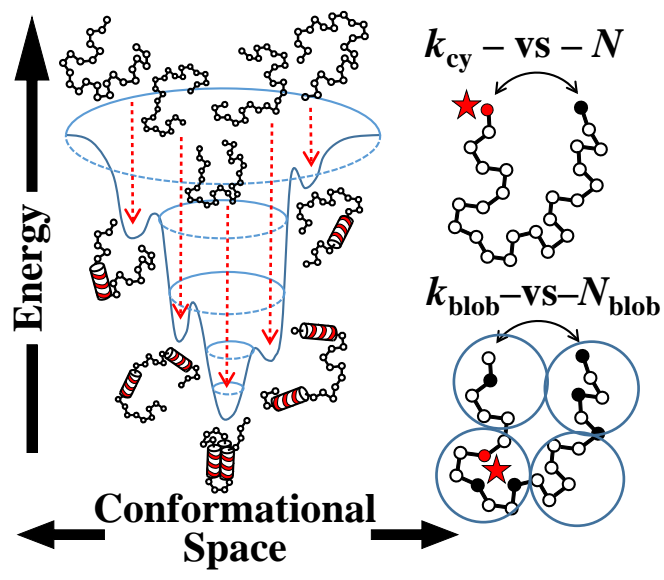

Biography

Shiva Farhangi is finishing her $\mathrm{PhD}$ thesis in polymer chemistry under the supervision of Prof. Jean Duhamel at the University of Waterloo. Her PhD work involved the synthesis of poly(alkyl methacrylate)s randomly labeled with pyrene derivatives and their characterization by fluorescence. While waiting for her $\mathrm{PhD}$ defense, she started a post-doctorate fellowship in medicinal chemistry at the University of Toronto in Mississauga under the supervision of Dr. Gunning's. Her current work aims to characterize molecular associations between XXX events using a wide variety of biophysical techniques such as SPR, fluorescence, and ITC.

Biography

After being trained as a chemical engineer from the Ecole Nationale Supérieure des Industries Chimiques in Nancy (France), Jean Duhamel studied the diffusional effects observed during excimer formation in viscous solvents for his $\mathrm{PhD}$ thesis which he obtained in 1989 under the supervision of Dr. André at the Institut National Polytechnique de Lorraine (France). Following a first post-doc at the U. of Toronto (Canada) with Prof. Winnik and a second one at the U. of Pennsylvania (USA) with Prof. Lu, he joined the U. of Waterloo in 1996. Work from his research group has established that pyrene excimer fluorescence or PEF can be applied to characterize the internal dynamics of any macromolecule in solution.

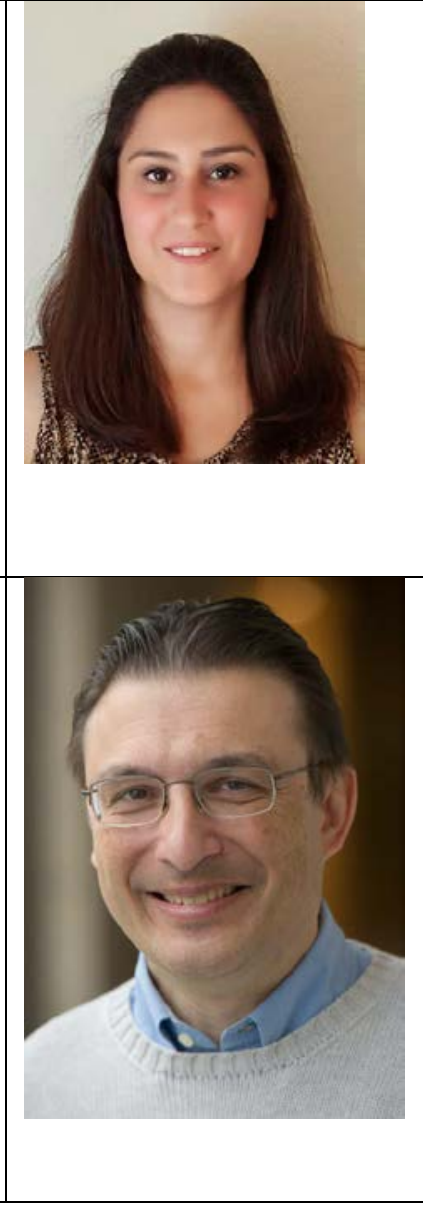

